ESAIM: M2AN

Vol. 40, No 6, 2006, pp. 1069-1100

DOI: $10.1051 / \mathrm{m} 2 \mathrm{an}: 2007001$
ESAIM: Mathematical Modelling and Numerical Analysis

www.edpsciences.org/m2an

\title{
FINITE VOLUME SCHEMES FOR FULLY NON-LINEAR ELLIPTIC EQUATIONS IN DIVERGENCE FORM
}

\author{
JÉRÔME DRONIOU ${ }^{1}$
}

\begin{abstract}
We construct finite volume schemes, on unstructured and irregular grids and in any space dimension, for non-linear elliptic equations of the $p$-Laplacian kind: $-\operatorname{div}\left(|\nabla u|^{p-2} \nabla u\right)=f$ (with $1<p<\infty)$. We prove the existence and uniqueness of the approximate solutions, as well as their strong convergence towards the solution of the PDE. The outcome of some numerical tests are also provided.
\end{abstract}

Mathematics Subject Classification. 65N12, 35J65, 65N30.

Received: February 2, 2006.

\section{INTRODUCTION}

We consider non-linear elliptic equations of the Leray-Lions kind:

$$
\begin{cases}-\operatorname{div}(a(x, \nabla \bar{u}))=f & \text { in } \Omega, \\ \bar{u}=0 & \text { on } \partial \Omega,\end{cases}
$$

where $\Omega$ is a bounded open polygonal subset of $\mathbb{R}^{d}$ and, for some $\left.p \in\right] 1, \infty[$,

$$
\begin{gathered}
a: \Omega \times \mathbb{R}^{d} \rightarrow \mathbb{R}^{d} \text { is a Caratheodory function (i.e. measurable w.r.t. its first variable } \\
\text { and continuous w.r.t. its second variable), } \\
\exists \alpha_{0}>0 \text { such that } a(x, \xi) \cdot \xi \geq \alpha_{0}|\xi|^{p} \text { for a.e. } x \in \Omega \text { and all } \xi \in \mathbb{R}^{d}, \\
(a(x, \xi)-a(x, \eta)) \cdot(\xi-\eta)>0 \text { for a.e. } x \in \Omega \text { and all } \xi \neq \eta, \\
\exists b \in L^{p^{\prime}}(\Omega), \quad \exists \Lambda>0 \text { such that }|a(x, \xi)| \leq b(x)+\Lambda|\xi|^{p-1} \text { for a.e. } x \in \Omega \text { and all } \xi \in \mathbb{R}^{d}, \\
f\left(\in L^{p^{\prime}}(\Omega) .\right.
\end{gathered}
$$

It is known, see [22], that such equations have unique weak solutions in $W_{0}^{1, p}(\Omega)$ (the uniqueness comes from the fact that we consider a monotone operator: $a$ does not depend on $\bar{u}$ ). These kinds of problem appear for example in the motion of glaciers [21], in flows of incompressible turbulent fluids through porous media [11] or in airfoil design [20]. They also serve as basic references for the mathematical study of fully non-linear elliptic equations (the canonical example being the $p$-Laplacian: $-\operatorname{div}\left(|\nabla u|^{p-2} \nabla u\right)=f$ ).

Keywords and phrases. Finite volume schemes, irregular grids, non-linear elliptic equations, Leray-Lions operators.

1 Département de Mathématiques, UMR CNRS 5149, CC 051, Université Montpellier II, Place Eugène Bataillon, 34095 Montpellier cedex 5, France. droniou@math.univ-montp2.fr

(c) EDP Sciences, SMAI 2007 
Finite element approximation of (1.1) has been studied in a number of papers, such as $[5,8,16-18,21,24]$. These references give error estimates on the approximation, but are mainly restricted to the case of two-dimensional domains $\Omega$ and/or to problems that come from the minimization of a functional.

Since (1.1) appears in physical models, it seems natural to try and approximate it with schemes which preserve physical properties; finite volume methods are among such schemes (they preserve the conservativity of the fluxes, for example). Their principle is to integrate the PDE in (1.1) on small polygonal sets inside $\Omega$ (the control volumes); using Stokes formula, this gives an equation on the fluxes of $a(\nabla \bar{u})$ through the edges of each control volume. One must then approximate these fluxes, using for example values of $\bar{u}$ on the control volumes on each side of the edges; this leads to a system on these values, which is the finite volume scheme. For linear equations, say $a(\nabla \bar{u})=\nabla \bar{u}$, approximating the flux $\int_{\sigma} \nabla \bar{u} \cdot \mathbf{n}$ through an edge $\sigma$ (n is a unit normal to $\left.\sigma\right)$ only demands to approximate the normal component $\nabla \bar{u} \cdot \mathbf{n}$ of $\nabla \bar{u}$, which can be easily done thanks to the values of $\bar{u}$ on each side of $\sigma$. But for non-linear equations, for example $a(\nabla \bar{u})=|\nabla \bar{u}|^{p-2} \nabla \bar{u}$, approximating the flux $\int_{\sigma}|\nabla \bar{u}|^{p-2} \nabla \bar{u} \cdot \mathbf{n}$ demands to approximate all the components of $\nabla \bar{u}$ (because of the term $|\nabla \bar{u}|^{p-2}$ ), which is far less easy to do.

In a series of papers, Andreianov et al. construct finite volume schemes for (1.1), at first for the $p$-Laplacian on cartesian grids [2,3] and, more recently, for Leray-Lions operators on general meshes [4]. To approximate the whole gradient $\nabla \bar{u}$ with values of $\bar{u}$, they use dual meshes (two grids on $\Omega$ ) and either a four-point finite difference method (on cartesian grids) or the gradient reconstruction introduced in [9] (on general grids). They show the convergence of their schemes and, under additional hypotheses on $a$, fine error estimates (using optimal regularity results for the solution). However, these schemes are presented on two-dimensional domains and their extension to the case $d \geq 3$ does not seem straightforward, considering the difficulty of manipulating dual meshes in higher dimensions.

The idea of the mixed finite volume scheme we use is to keep the fluxes of $a(\nabla \bar{u})$ and the gradient of $\bar{u}$ as unknowns, and not to try, in a first time, to approximate these fluxes and gradient using the values of $\bar{u}$. The integration of the PDE on each control volume gives an equation on the fluxes, with which we reconstruct $a(\nabla \bar{u})$ thanks to a general formula (see Lem. 8.2). We thus write a quite simple scheme which not only handles fully non-linear equations but can also be applied in any space dimension and to a wide variety of grids on $\Omega$ (see Def. 2.1).

In the following section, we present the finite volume scheme for (1.1) and we state the main results (existence, uniqueness and convergence of the approximate solutions). Our scheme is based on three unknowns functions $(u, \mathbf{v}, F)$, which respectively correspond to approximations of $\bar{u}$, of $\nabla \bar{u}$ and of the fluxes of $a(\nabla \bar{u})$; one of the equations quite naturally states that $\mathbf{v}$ is, in a sense, the gradient of $u$ (with a penalization involving $F$ ): in Section 3 , we give some basic properties satisfied by the $(u, \mathbf{v}, F)$ which verify this particular equation. Section 4 is devoted to the proof of the results stated in Section 2. In Section 5, we study some generalizations of the scheme presented in Section 2: a scheme for non-monotone operators (that is to say, equations of the kind $-\operatorname{div}(a(x, \bar{u}, \nabla \bar{u}))=f)$, a non-penalized scheme, and a scheme for right-hand sides in $W^{-1, p^{\prime}}(\Omega)$. We have run numerical experiments, and we present some of their results in Section 6. After a short conclusion (Sect. 7), an appendix (Sect. 8) gathers a few technical results used in the paper.

\section{The Finite Volume DiscRetization}

Our finite volume scheme for (1.1) is inspired by the mixed finite volume scheme introduced in [12]. Let us first recall the notion of admissible discretization of $\Omega$.

Definition 2.1 (admissible discretization). Let $\Omega$ be an open bounded polygonal subset of $\mathbb{R}^{d}$. An admissible finite volume discretization of $\Omega$ is given by $\mathcal{D}=(\mathcal{M}, \mathcal{E}, \mathcal{P})$, where:

- $\mathcal{M}$ is a finite family of non empty open polygonal convex disjoint subsets of $\Omega$ (the "control volumes") such that $\bar{\Omega}=\cup_{K \in \mathcal{M}} \bar{K}$.

- $\mathcal{E}$ is a finite family of disjoint subsets of $\bar{\Omega}$ (the "edges" of the mesh), such that, for all $\sigma \in \mathcal{E}$, there exists an affine hyperplane $E$ of $\mathbb{R}^{d}$ and $K \in \mathcal{M}$ verifying: $\sigma \subset \partial K \cap E$ and $\sigma$ is a non empty open convex 
subset of $E$. We assume that, for all $K \in \mathcal{M}$, there exists a subset $\mathcal{E}_{K}$ of $\mathcal{E}$ such that $\partial K=\cup_{\sigma \in \mathcal{E}_{K}} \bar{\sigma}$. We also assume that, for all $\sigma \in \mathcal{E}$, either $\sigma \subset \partial \Omega$ or $\bar{\sigma}=\bar{K} \cap \bar{L}$ for some $(K, L) \in \mathcal{M}^{2}$.

- $\mathcal{P}$ is a family of points of $\Omega$ indexed by $\mathcal{M}$, denoted by $\mathcal{P}=\left(\mathbf{x}_{K}\right)_{K \in \mathcal{M}}$ and such that, for all $K \in \mathcal{M}$, $\mathbf{x}_{K} \in K$.

Remark 2.1. This definition of discretization allows a wide variety of grids, in any space dimension and with very few geometric restrictions. In particular, we accept meshes which are not admissible with respect to the definition given in [15], and meshes whose edges have been cut in two (or more) via a local refinement procedure. See [12].

Also, it is not really mandatory that each $\mathbf{x}_{K}$ be in its control volume: it only needs to stay within distance $\sim \operatorname{diam}(K)$ of $K$.

In the rest of the paper, we use the following notations associated with an admissible discretization $\mathcal{D}$. The $d$-dimensional measure of a control volume $K$ is written $\mathrm{m}(K)$, and the $(d-1)$-dimensional measure of an edge $\sigma$ is $\mathrm{m}(\sigma)$; in the integral signs, $\gamma$ denotes the measure on the edges. If $\sigma \in \mathcal{E}_{K}$, then $\mathbf{n}_{K, \sigma}$ is the unit normal to $\sigma$ outward to $K$. In the case where $\sigma \in \mathcal{E}$ is such that $\bar{\sigma}=\bar{K} \cap \bar{L}$ for $(K, L) \in \mathcal{M}^{2}$, we denote $\sigma=K \mid L$. For all $\sigma \in \mathcal{E}, \mathbf{x}_{\sigma}$ is the barycenter of $\sigma$. The set of interior (resp. boundary) edges is defined as $\mathcal{E}_{\text {int }}=\{\sigma \in \mathcal{E}$; $\sigma \not \subset \partial \Omega\}$ (resp. $\mathcal{E}_{\text {ext }}=\{\sigma \in \mathcal{E} ; \sigma \subset \partial \Omega\}$ ).

We study the convergence of the approximations as the size of the discretization

$$
\operatorname{size}(\mathcal{D})=\sup \{\operatorname{diam}(K) ; K \in \mathcal{M}\}
$$

tends to 0 , under the assumption that the regularity of the discretization stays bounded, the regularity being defined by

$$
\operatorname{regul}(\mathcal{D})=\sup \left\{\max \left(\frac{\operatorname{diam}(K)^{d}}{\rho_{K}^{d}}, \operatorname{Card}\left(\mathcal{E}_{K}\right)\right) ; K \in \mathcal{M}\right\}
$$

where, for $K \in \mathcal{M}, \rho_{K}$ is the supremum of the radius of the balls contained in $K$ (notice that $\operatorname{regul}(\mathcal{D})$ stays bounded under a local refinement procedure). One of the main interests of this quantity is the following inequality: for all $K \in \mathcal{M}$,

$$
\operatorname{diam}(K)^{d} \leq \operatorname{regul}(\mathcal{D}) \rho_{K}^{d} \leq \frac{\operatorname{regul}(\mathcal{D})}{\omega_{d}} \mathrm{~m}(K)
$$

where $\omega_{d}$ is the volume of the unit ball in $\mathbb{R}^{d}$.

If $\mathcal{D}$ is an admissible discretization of $\Omega$, we denote by $H_{\mathcal{D}}$ the space of functions $\Omega \rightarrow \mathbb{R}$ which are constant on each control volume $K \in \mathcal{M}$ (the value on $K$ of a function $g \in H_{\mathcal{D}}$ is written $g_{K}$ ), and by $\mathcal{F}$ the space of

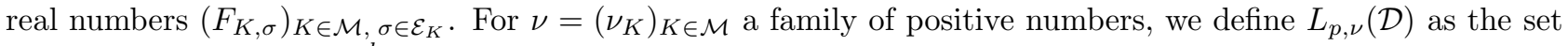
of all $(u, \mathbf{v}, F) \in H_{\mathcal{D}} \times H_{\mathcal{D}}^{d} \times \mathcal{F}$ such that

$$
\begin{gathered}
\mathbf{v}_{K} \cdot\left(\mathbf{x}_{\sigma}-\mathbf{x}_{K}\right)+\mathbf{v}_{L} \cdot\left(\mathbf{x}_{L}-\mathbf{x}_{\sigma}\right)+\nu_{K} \mathrm{~m}(K)\left|F_{K, \sigma}\right|^{\frac{1}{p-1}-1} F_{K, \sigma}-\nu_{L} \mathrm{~m}(L)\left|F_{L, \sigma}\right|^{\frac{1}{p-1}-1} F_{L, \sigma} \\
=u_{L}-u_{K}, \quad \forall \sigma=K \mid L \in \mathcal{E}_{\text {int }}, \\
\mathbf{v}_{K} \cdot\left(\mathbf{x}_{\sigma}-\mathbf{x}_{K}\right)+\nu_{K} \mathrm{~m}(K)\left|F_{K, \sigma}\right|^{\frac{1}{p-1}-1} F_{K, \sigma}=-u_{K}, \quad \forall K \in \mathcal{M}, \forall \sigma \in \mathcal{E}_{K} \cap \mathcal{E}_{\text {ext }} .
\end{gathered}
$$

We let $a_{K}(\xi)=\frac{1}{\mathrm{~m}(K)} \int_{K} a(x, \xi) \mathrm{d} x$ and we consider the following finite volume scheme for $(1.1)$ : find $(u, \mathbf{v}, F) \in$ $L_{p, \nu}(\mathcal{D})$ such that

$$
\begin{gathered}
F_{K, \sigma}+F_{L, \sigma}=0, \quad \forall \sigma=K \mid L \in \mathcal{E}_{\mathrm{int}}, \\
\mathrm{m}(K) a_{K}\left(\mathbf{v}_{K}\right)=\sum_{\sigma \in \mathcal{E}_{K}} F_{K, \sigma}\left(\mathbf{x}_{\sigma}-\mathbf{x}_{K}\right), \quad \forall K \in \mathcal{M}, \\
-\sum_{\sigma \in \mathcal{E}_{K}} F_{K, \sigma}=\int_{K} f(x) \mathrm{d} x, \quad \forall K \in \mathcal{M} .
\end{gathered}
$$


The scheme $((2.2),(2.3),(2.4),(2.5))$ is quite easy to understand if we point out that $u$ and $\mathbf{v}$ play the role of approximations of $\bar{u}$ (the solution to (1.1)) and $\nabla \bar{u}$. Equation (2.5) comes from a formal integration of (1.1) on each control volume $K, F_{K, \sigma}$ being an approximation of the flux of $a(\nabla \bar{u})$ through $\sigma: F_{K, \sigma} \approx \int_{\sigma} a(\nabla \bar{u}) \cdot \mathbf{n}_{K, \sigma} \mathrm{d} \gamma$. This expression shows that these fluxes are naturally conservative, hence (2.3). Lemma 8.2 in the appendix gives a formula which reconstructs a vector knowing its fluxes on the edges of a control volume: (2.4) simply states that we use this formula to reconstruct $a(\nabla \bar{u})$ (recall that $\mathbf{v}_{K}$ is an approximation on $K$ of $\nabla \bar{u}$ ). Lastly, (2.2) is the expression (if we forget the fluxes) that $\nabla \bar{u}$ (approximated by $\mathbf{v}$ ) is the gradient of $\bar{u}$ (approximated by $u$ ); we need to penalize this equation with the terms $\left|F_{K, \sigma}\right|^{\frac{1}{p-1}-1} F_{K, \sigma}$ in order to estimate the fluxes and ensure their uniqueness (see also Subsect. 5.2); to understand the power chosen for $F_{K, \sigma}$, we can consider the case of the $p$-Laplacian $a(\nabla \bar{u})=|\nabla \bar{u}|^{p-2} \nabla \bar{u}$ : in this situation, $\left|F_{K, \sigma}\right| \approx \mathrm{m}(\sigma)|\nabla \bar{u}|^{p-1}$ and, since $\left|\mathbf{v}_{K}\right| \approx|\nabla \bar{u}|$, it is quite natural that $F_{K, \sigma}$ appears with total power equal to $\frac{1}{p-1}$.

The main results of this paper are the following two theorems. The first one states that the finite volume scheme has a unique solution, and the second one that this solution converges, as the size of the discretization tends to 0 , to the weak solution of (1.1).

Theorem 2.1. Under Hypotheses (1.2)-(1.6), if $\mathcal{D}$ is an admissible discretization of $\Omega$ and $\left(\nu_{K}\right)_{K \in \mathcal{M}}$ is a family of positive numbers, then there exists a unique solution $(u, \mathbf{v}, F)$ to $((2.2)-(2.5))$.

Theorem 2.2. Assume Hypotheses (1.2)-(1.6). Let $\left(\mathcal{D}_{n}\right)_{n \geq 1}$ be a sequence of admissible discretizations of $\Omega$ such that $\operatorname{size}\left(\mathcal{D}_{n}\right) \rightarrow 0$ and $\left(\operatorname{regul}\left(\mathcal{D}_{n}\right)\right)_{n \geq 1}$ is bounded. Let $\nu_{0}>0$ and $\left.\beta \in\right]-p^{\prime}(d-1),-p^{\prime}(d-2)[$. Let $\left(u_{n}, \mathbf{v}_{n}, F_{n}\right)$ be the solution to $((2.2)-(2.5))$ for $\mathcal{D}=\mathcal{D}_{n}$ and $\nu_{K}=\nu_{0} \operatorname{diam}(K)^{\beta}$ for all $K \in \mathcal{M}_{n}$. Let $\bar{u} \in W_{0}^{1, p}(\Omega)$ be the weak solution to (1.1).

Then, as $n \rightarrow \infty, u_{n} \rightarrow \bar{u}$ weakly in $L^{p}(\Omega)$ and strongly in $L^{q}(\Omega)$ for all $q<p$, and $\mathbf{v}_{n} \rightarrow \nabla \bar{u}$ strongly in $L^{p}(\Omega)^{d}$.

Remark 2.2. By (1.2) and (1.5), the strong convergence of $\mathbf{v}_{n}$ to $\nabla \bar{u}$ in $L^{p}(\Omega)^{d}$ implies the strong convergence of $a\left(\cdot, \mathbf{v}_{n}\right)$ to $a(\cdot, \nabla \bar{u})$ in $L^{p^{\prime}}(\Omega)^{d}$.

Remark 2.3. If the "> in (1.4) is replaced by " $\geq$ ", these results hold with the following changes: there is not uniqueness of the solution to the approximate (or limit) problem, the convergence holds only up to a subsequence, and the convergence of $\mathbf{v}_{n}$ is weak in $L^{p}(\Omega)^{d}$.

\section{Properties of $L_{p, \nu}(\mathcal{D})$}

To study the convergence of the scheme, we need some properties of the set $L_{p, \nu}(\mathcal{D})$. In the case $p=2$, the following lemmas are proved in [12]; for the sake of completeness, we give below the full proofs for any $p$, using and even simplifying [12] whenever possible.

Lemma 3.1 (Poincaré's inequality). Let $\mathcal{D}$ be an admissible discretization of $\Omega$ such that regul $(\mathcal{D}) \leq \theta$ for some $\theta>0$, and let $\left(\nu_{K}\right)_{K \in \mathcal{M}}$ be a family of positive real numbers. There exists $C_{1}$ only depending on $d, p, \Omega$ and $\theta$ such that, for all $(u, \mathbf{v}, F) \in L_{p, \nu}(\mathcal{D})$,

$$
\|u\|_{L^{p}(\Omega)} \leq C_{1}\left(\|\mathbf{v}\|_{L^{p}(\Omega)^{d}}+M_{p}(\mathcal{D}, \nu, F)\right),
$$

where $M_{p}(\mathcal{D}, \nu, F)=\left(\sum_{K \in \mathcal{M}} \sum_{\sigma \in \mathcal{E}_{K}} \operatorname{diam}(K)^{(d-1) p} \nu_{K}^{p}\left(\left|F_{K, \sigma}\right|^{\frac{1}{p-1}}\right)^{p} \mathrm{~m}(K)\right)^{\frac{1}{p}}$.

Proof of Lemma 3.1. Let $B$ be a ball containing $\Omega$ and let $w$ be the weak solution of $-\Delta w=|u|^{p-2} u$ on $B$ with value 0 on $\partial B$ (we have extended $u$ by 0 outside $\Omega$ ). By well known regularity results (see e.g. $[1,23]$ ), there exists $C_{2}$ only depending on $d, B$ and $p$ (that is, on $d, \Omega$ and $p$ ) such that $w \in W^{2, p^{\prime}}(B)$ with

$$
\|w\|_{W^{2, p^{\prime}}(B)} \leq C_{2}\left\||u|^{p-2} u\right\|_{L^{p^{\prime}(B)}}=C_{2}\|u\|_{L^{p}(\Omega)}^{p-1} .
$$


We multiply each equation of (2.2) by $\int_{\sigma} \nabla w \cdot \mathbf{n}_{K, \sigma} \mathrm{d} \gamma$, sum over the edges and gather by control volumes using $\mathbf{n}_{K, \sigma}=-\mathbf{n}_{L, \sigma}$ whenever $\sigma=K \mid L$; this gives

$$
\begin{aligned}
\sum_{K \in \mathcal{M}} \sum_{\sigma \in \mathcal{E}_{K}} \mathbf{v}_{K} \cdot\left(\mathbf{x}_{\sigma}-\mathbf{x}_{K}\right) \int_{\sigma} \nabla w \cdot \mathbf{n}_{K, \sigma} \mathrm{d} \gamma & +\sum_{K \in \mathcal{M}} \sum_{\sigma \in \mathcal{E}_{K}} \nu_{K} \mathrm{~m}(K)\left|F_{K, \sigma}\right|^{\frac{1}{p-1}-1} F_{K, \sigma} \int_{\sigma} \nabla w \cdot \mathbf{n}_{K, \sigma} \mathrm{d} \gamma \\
& =-\sum_{K \in \mathcal{M}} u_{K} \sum_{\sigma \in \mathcal{E}_{K}} \int_{\sigma} \nabla w \cdot \mathbf{n}_{K, \sigma} \mathrm{d} \gamma \\
& =-\sum_{K \in \mathcal{M}} u_{K} \int_{K} \Delta w(x) \mathrm{d} x \\
& =\|u\|_{L^{p}(\Omega)}^{p}
\end{aligned}
$$

Let us denote $T_{1}$ and $T_{2}$ the two terms in the left-hand side of this equality.

Since $\operatorname{regul}(\mathcal{D}) \leq \theta$, we can apply Lemma 8.1 to find $C_{3}$ only depending on $d, p, \Omega$ and $\theta$ such that

$$
\left|\frac{1}{\mathrm{~m}(\sigma)} \int_{\sigma} \nabla w \mathrm{~d} \gamma \cdot \mathbf{n}_{K, \sigma}\right|^{p^{\prime}} \leq\left|\frac{1}{\mathrm{~m}(\sigma)} \int_{\sigma} \nabla w \mathrm{~d} \gamma\right|^{p^{\prime}} \leq \frac{C_{3}}{\mathrm{~m}(\sigma) \operatorname{diam}(K)}\|w\|_{W^{2, p^{\prime}}(K)}^{p^{\prime}}
$$

(we have bounded $\operatorname{diam}(K)^{p^{\prime}}$, which appears when applying Lemma 8.1, by $\operatorname{diam}(\Omega)^{p^{\prime}}$ ). Therefore, for all real numbers $\lambda_{K, \sigma}$, by Hölder's inequality,

$$
\begin{aligned}
&\left|\sum_{K \in \mathcal{M}} \sum_{\sigma \in \mathcal{E}_{K}} \lambda_{K, \sigma} \int_{\sigma} \nabla w \cdot \mathbf{n}_{K, \sigma} \mathrm{d} \gamma\right| \\
&=\left|\sum_{K \in \mathcal{M}} \sum_{\sigma \in \mathcal{E}_{K}} \mathrm{~m}(\sigma)\left(\operatorname{diam}(K)^{-\frac{1}{p^{\prime}}} \lambda_{K, \sigma}\right) \times\left(\operatorname{diam}(K)^{\frac{1}{p^{\prime}}} \frac{1}{\mathrm{~m}(\sigma)} \int_{\sigma} \nabla w \mathrm{~d} \gamma \cdot \mathbf{n}_{K, \sigma}\right)\right| \\
& \leq\left(\sum_{K \in \mathcal{M}} \sum_{\sigma \in \mathcal{E}_{K}} \mathrm{~m}(\sigma)\left|\operatorname{diam}(K)^{-\frac{1}{p^{\prime}}} \lambda_{K, \sigma}\right|^{p}\right)^{\frac{1}{p}} \\
& \times\left(\sum_{K \in \mathcal{M}} \sum_{\sigma \in \mathcal{E}_{K}} \mathrm{~m}(\sigma)\left|\operatorname{diam}(K)^{\frac{1}{p^{\prime}}} \frac{1}{\mathrm{~m}(\sigma)} \int_{\sigma} \nabla w \mathrm{~d} \gamma \cdot \mathbf{n}_{K, \sigma}\right|^{p^{\prime}}\right)^{\frac{1}{p^{\prime}}} \\
& \leq\left(\sum_{K \in \mathcal{M}} \sum_{\sigma \in \mathcal{E}_{K}} \mathrm{~m}(\sigma) \operatorname{diam}(K)^{-(p-1)}\left|\lambda_{K, \sigma}\right|^{p}\right)^{\frac{1}{p}}\left(\sum_{K \in \mathcal{M}} \sum_{\sigma \in \mathcal{E}_{K}} C_{3}\|w\|_{W^{2, p^{\prime}}(K)}^{p^{\prime}}\right)^{\frac{1}{p^{\prime}}} \\
& \leq\left(\omega_{d-1} \sum_{K \in \mathcal{M}} \sum_{\sigma \in \mathcal{E}_{K}} \operatorname{diam}(K)^{d-p}\left|\lambda_{K, \sigma}\right|^{p}\right)^{\frac{1}{p}} C_{3}^{\frac{1}{p^{\prime}}} \operatorname{regul}(\mathcal{D})^{\frac{1}{p^{\prime}}}\|w\|_{W^{2, p^{\prime}}(\Omega)}
\end{aligned}
$$

(we have used the fact that $\operatorname{Card}\left(\mathcal{E}_{K}\right) \leq \operatorname{regul}(\mathcal{D})$ and that, if $\sigma \in \mathcal{E}_{K}$, then $\mathrm{m}(\sigma) \leq \omega_{d-1} \operatorname{diam}(K)^{d-1}$ since $\operatorname{diam}(\sigma) \leq \operatorname{diam}(K))$. Applying this estimate to $\lambda_{K, \sigma}=\mathbf{v}_{K} \cdot\left(\mathbf{x}_{\sigma}-\mathbf{x}_{K}\right)$ we find, thanks to (2.1) and 
since $\operatorname{regul}(\mathcal{D}) \leq \theta$,

$$
\begin{aligned}
\left|T_{1}\right| & \leq C_{3}^{\frac{1}{p^{\prime}}} \theta^{\frac{1}{p^{\prime}}}|| w \|_{W^{2, p^{\prime}}(\Omega)}\left(\omega_{d-1} \sum_{K \in \mathcal{M}} \sum_{\sigma \in \mathcal{E}_{K}} \operatorname{diam}(K)^{d-p}\left|\mathbf{v}_{K}\right|^{p} \operatorname{diam}(K)^{p}\right)^{\frac{1}{p}} \\
& \leq C_{3}^{\frac{1}{p^{\prime}}} \theta^{\frac{1}{p^{\prime}}}\|w\|_{W^{2, p^{\prime}}(\Omega)}\left(\frac{\operatorname{regul}(\mathcal{D})^{2} \omega_{d-1}}{\omega_{d}} \sum_{K \in \mathcal{M}} \mathrm{m}(K)\left|\mathbf{v}_{K}\right|^{p}\right)^{\frac{1}{p}} \\
& \leq C_{3}^{\frac{1}{p^{\prime}}} \theta^{\frac{1}{p^{\prime}}}\|w\|_{W^{2, p^{\prime}}(\Omega)}\left(\frac{\theta^{2} \omega_{d-1}}{\omega_{d}}\right)^{\frac{1}{p}}\|\mathbf{v}\|_{L^{p}(\Omega)^{d}}
\end{aligned}
$$

and, with $\lambda_{K, \sigma}=\nu_{K} \mathrm{~m}(K)\left|F_{K, \sigma}\right|^{\frac{1}{p-1}-1} F_{K, \sigma}$, since $\mathrm{m}(K) \leq \omega_{d} \operatorname{diam}(K)^{d}$,

$$
\begin{aligned}
\left|T_{2}\right| \leq & C_{3}^{\frac{1}{p^{p}}} \theta^{\frac{1}{p^{\prime}}}|| w \|_{W^{2, p^{\prime}}(\Omega)}\left(\omega_{d-1} \sum_{K \in \mathcal{M}} \sum_{\sigma \in \mathcal{E}_{K}} \operatorname{diam}(K)^{d-p} \nu_{K}^{p} \mathrm{~m}(K)^{p}\left(\left|F_{K, \sigma}\right|^{\frac{1}{p-1}}\right)^{p}\right)^{\frac{1}{p}} \\
\leq & C_{3}^{\frac{1}{p^{\prime}}} \theta^{\frac{1}{p^{\prime}}}|| w \|_{W^{2, p^{\prime}}(\Omega)} \\
& \times\left(\omega_{d-1} \sum_{K \in \mathcal{M}} \sum_{\sigma \in \mathcal{E}_{K}} \operatorname{diam}(K)^{d-p} \omega_{d}^{p-1} \operatorname{diam}(K)^{d(p-1)} \mathrm{m}(K) \nu_{K}^{p}\left(\left|F_{K, \sigma}\right|^{\frac{1}{p-1}}\right)^{p}\right)^{\frac{1}{p}} \\
\leq & C_{3}^{\frac{1}{p^{p}}} \theta^{\frac{1}{p^{\prime}}}|| w \|_{W^{2, p^{\prime}}(\Omega)}\left(\omega_{d-1} \omega_{d}^{p-1} \sum_{K \in \mathcal{M}} \sum_{\sigma \in \mathcal{E}_{K}} \operatorname{diam}(K)^{(d-1) p} \nu_{K}^{p}\left(\left|F_{K, \sigma}\right|^{\frac{1}{p-1}}\right)^{p} \mathrm{~m}(K)\right)^{\frac{1}{p}} .
\end{aligned}
$$

Gathering (3.2), (3.4) and (3.5), we see that

$$
\|u\|_{L^{p}(\Omega)}^{p} \leq C_{4}\|w\|_{W^{2, p^{\prime}}(\Omega)}\|\mathbf{v}\|_{L^{p}(\Omega)^{d}}+C_{4}\|w\|_{W^{2, p^{\prime}}(\Omega)} M_{p}(\mathcal{D}, \nu, F)
$$

for some $C_{4}$ only depending on $d, p, \Omega$ and $\theta$, and we conclude the proof by (3.1).

Lemma 3.2 (equicontinuity of the translations). Let $\mathcal{D}$ be an admissible discretization of $\Omega$ such that regul $(\mathcal{D}) \leq$ $\theta$ for some $\theta>0$, and let $\left(\nu_{K}\right)_{K \in \mathcal{M}}$ be a family of positive real numbers. There exists $C_{5}$ only depending on $\bar{d}$, $\Omega$ and $\theta$ such that, for all $(u, \mathbf{v}, F) \in L_{p, \nu}(\mathcal{D})$ and all $\xi \in \mathbb{R}^{d}$,

$$
\|u(\cdot+\xi)-u\|_{L^{1}\left(\mathbb{R}^{d}\right)} \leq C_{5}\left(\|\mathbf{v}\|_{L^{1}(\Omega)^{d}}+M_{1}(\mathcal{D}, \nu, F)\right)|\xi|,
$$

where $M_{1}(\mathcal{D}, \nu, F)=\sum_{K \in \mathcal{M}} \sum_{\sigma \in \mathcal{E}_{K}} \operatorname{diam}(K)^{d-1} \nu_{K}\left|F_{K, \sigma}\right|^{\frac{1}{p-1}} \mathrm{~m}(K)$ and $u$ has been extended by 0 outside $\Omega$.

Proof of Lemma 3.2. Let $\widetilde{F}_{K, \sigma}=\left|F_{K, \sigma}\right|^{\frac{1}{p-1}-1} F_{K, \sigma}$. If $(u, \mathbf{v}, F) \in L_{p, \nu}(\mathcal{D})$, then $(u, \mathbf{v}, \widetilde{F}) \in L_{2, \nu}(\mathcal{D})$ and we can apply Lemma 3.2 in [12] (which is in fact exactly the result we want to prove in the case $p=2$ ): there exists $C_{5}$ only depending on $d, \Omega$ and $\theta$ such that, for all $\xi \in \mathbb{R}^{d}$,

$$
\|u(\cdot+\xi)-u\|_{L^{1}\left(\mathbb{R}^{d}\right)} \leq C_{5}\left(\|\mathbf{v}\|_{L^{1}(\Omega)^{d}}+\sum_{K \in \mathcal{M}} \sum_{\sigma \in \mathcal{E}_{K}} \operatorname{diam}(K)^{d-1} \nu_{K}\left|\widetilde{F}_{K, \sigma}\right| \mathrm{m}(K)\right)|\xi| .
$$

By definition of $\widetilde{F}_{K, \sigma}$, this concludes the proof. 
Lemma 3.3 (compactness property). Let $\left(\mathcal{D}_{n}\right)_{n \geq 1}$ be a sequence of admissible discretizations of $\Omega$ such that $\operatorname{size}\left(\mathcal{D}_{n}\right) \rightarrow 0$ and $\left(\operatorname{regul}\left(\mathcal{D}_{n}\right)\right)_{n>1}$ is bounded. Let $\left(\nu_{n}\right)_{n>1}$ be a sequence of families of positive numbers (each family being indexed by $\left.\mathcal{M}_{n}\right)$. We take $\left(u_{n}, \mathbf{v}_{n}, F_{n}\right) \in L_{p, \nu_{n}}\left(\mathcal{D}_{n}\right)$ and we assume that $\left(\left\|\mathbf{v}_{n}\right\|_{L^{p}(\Omega)^{d}}\right)_{n \geq 1}$ and $\left(M_{p}\left(\mathcal{D}_{n}, \nu_{n}, F_{n}\right)\right)_{n \geq 1}$ are bounded, and that $M_{1}\left(\mathcal{D}_{n}, \nu_{n}, F_{n}\right) \rightarrow 0$ as $n \rightarrow \infty\left(M_{p}\right.$ and $M_{1}$ are defined in Lems. 3.1 and 3.2$)$.

Then there exists $\bar{u} \in W_{0}^{1, p}(\Omega)$ such that, up to a subsequence, $u_{n} \rightarrow \bar{u}$ weakly in $L^{p}(\Omega)$ and strongly in $L^{q}(\Omega)$ for all $q<p$, and $\mathbf{v}_{n} \rightarrow \nabla \bar{u}$ weakly in $L^{p}(\Omega)^{d}$.

Proof of Lemma 3.3. Thanks to the assumptions, to Lemmas 3.1 and 3.2, and to Kolmogorov compactness theorem, we can assume that, up to a subsequence, $\left(u_{n}\right)_{n \geq 1}$ converges to some $\bar{u}$ weakly in $L^{p}(\Omega)$ and strongly in $L^{1}(\Omega)$, and that $\left(\mathbf{v}_{n}\right)_{n \geq 1}$ converges to some $\overline{\mathbf{v}}$ weakly in $L^{p}(\Omega)^{d}$. This implies $u_{n} \rightarrow \bar{u}$ strongly in $L^{q}(\Omega)$ for all $q<p$ and it remains to prove that $\bar{u} \in W_{0}^{1, p}(\Omega)$ and that $\overline{\mathbf{v}}=\nabla \bar{u}$.

To achieve this, we extend $\bar{u}$ and $\overline{\mathbf{v}}$ by 0 outside $\Omega$ and we prove that $\nabla \bar{u}=\overline{\mathbf{v}}$ in the distributional sense on $\mathbb{R}^{d}$ : this will in particular prove that $\bar{u} \in W^{1, p}\left(\mathbb{R}^{d}\right)$ and, since it is null outside $\Omega$, that it also belongs to $W_{0}^{1, p}(\Omega)$.

For simplicity of notations, we drop all the indices $n$. Let $\varphi \in C_{c}^{\infty}\left(\mathbb{R}^{d}\right)$ and $\mathbf{e} \in \mathbb{R}^{d}$; multiply each equation of (2.2) by $\int_{\sigma} \varphi \mathbf{e} \cdot \mathbf{n}_{K, \sigma} \mathrm{d} \gamma$, sum over the edges and gather by control volumes using $\mathbf{n}_{K, \sigma}=-\mathbf{n}_{L, \sigma}$ when $\sigma=K \mid L ;$ this leads to

$$
\begin{array}{r}
\sum_{K \in \mathcal{M}} \mathbf{v}_{K} \cdot \sum_{\sigma \in \mathcal{E}_{K}} \int_{\sigma} \varphi \mathbf{e} \cdot \mathbf{n}_{K, \sigma} \mathrm{d} \gamma\left(\mathbf{x}_{\sigma}-\mathbf{x}_{K}\right)+\sum_{K \in \mathcal{M}} \sum_{\sigma \in \mathcal{E}_{K}} \nu_{K} \mathrm{~m}(K)\left|F_{K, \sigma}\right|^{\frac{1}{p-1}-1} F_{K, \sigma} \int_{\sigma} \varphi \mathbf{e} \cdot \mathbf{n}_{K, \sigma} \mathrm{d} \gamma= \\
-\sum_{K \in \mathcal{M}} u_{K} \sum_{\sigma \in \mathcal{E}_{K}} \int_{\sigma} \varphi \mathbf{e} \cdot \mathbf{n}_{K, \sigma} \mathrm{d} \gamma
\end{array}
$$

Let $T_{3}, T_{4}$ and $T_{5}$ be the three terms of this equation.

We have

$$
\begin{aligned}
T_{5} & =-\sum_{K \in \mathcal{M}} u_{K} \int_{K} \operatorname{div}(\varphi \mathbf{e})(x) \mathrm{d} x \\
& =-\int_{\Omega} u(x) \operatorname{div}(\varphi \mathbf{e})(x) \mathrm{d} x \rightarrow-\int_{\Omega} \bar{u}(x) \operatorname{div}(\varphi \mathbf{e})(x) \mathrm{d} x \quad \text { as } \operatorname{size}(\mathcal{D}) \rightarrow 0 .
\end{aligned}
$$

Let

$$
T_{6}=\sum_{K \in \mathcal{M}} \mathbf{v}_{K} \cdot \sum_{\sigma \in \mathcal{E}_{K}} \mathrm{~m}(\sigma)\left(\frac{1}{\mathrm{~m}(K)} \int_{K} \varphi(x) \mathbf{e} \mathrm{d} x\right) \cdot \mathbf{n}_{K, \sigma}\left(\mathbf{x}_{\sigma}-\mathbf{x}_{K}\right) .
$$

We have, for all $\sigma \in \mathcal{E}_{K}, \mathrm{~m}(\sigma) \operatorname{diam}(K) \leq \omega_{d-1} \operatorname{diam}(K)^{d} \leq \frac{\omega_{d-1} \operatorname{regul}(\mathcal{D})}{\omega_{d}} \mathrm{~m}(K)$ (see (2.1)). Using the regularity of $\varphi$, we therefore obtain $C_{6}$ only depending on $\varphi$ such that

$$
\begin{aligned}
\left|T_{3}-T_{6}\right| & \leq C_{6} \sum_{K \in \mathcal{M}}\left|\mathbf{v}_{K}\right| \sum_{\sigma \in \mathcal{E}_{K}} \mathrm{~m}(\sigma) \operatorname{diam}(K)\left|\mathbf{x}_{\sigma}-\mathbf{x}_{K}\right| \\
& \leq C_{6} \operatorname{size}(\mathcal{D}) \sum_{K \in \mathcal{M}} \frac{\omega_{d-1} \operatorname{regul}(\mathcal{D})^{2}}{\omega_{d}} \mathrm{~m}(K)\left|\mathbf{v}_{K}\right| \\
& \leq C_{6} \operatorname{size}(\mathcal{D}) \frac{\omega_{d-1} \operatorname{regul}(\mathcal{D})^{2}}{\omega_{d}}\|\mathbf{v}\|_{L^{1}(\Omega)^{d}} \rightarrow 0 \quad \text { as } \operatorname{size}(\mathcal{D}) \rightarrow 0
\end{aligned}
$$


(we have used $\operatorname{Card}\left(\mathcal{E}_{K}\right) \leq \operatorname{regul}(\mathcal{D})$ ). Moreover, thanks to Lemma 8.2,

$$
T_{6}=\sum_{K \in \mathcal{M}} \mathbf{v}_{K} \cdot \int_{K} \varphi(x) \mathbf{e d} x=\int_{\Omega} \mathbf{v}(x) \cdot \varphi(x) \mathbf{e d} x \rightarrow \int_{\Omega} \overline{\mathbf{v}}(x) \cdot \varphi(x) \mathbf{e d} x \quad \text { as } \operatorname{size}(\mathcal{D}) \rightarrow 0 .
$$

Hence, $T_{3} \rightarrow \int_{\Omega} \overline{\mathbf{v}}(x) \cdot \varphi(x) \mathbf{e} \mathrm{d} x$ as $\operatorname{size}(\mathcal{D}) \rightarrow 0$.

By assumption,

$$
\left|T_{4}\right| \leq\|\varphi \mathbf{e}\|_{\infty} \sum_{K \in \mathcal{M}} \sum_{\sigma \in \mathcal{E}_{K}} \nu_{K} \mathrm{~m}(K)\left|F_{K, \sigma}\right|^{\frac{1}{p-1}} \mathrm{~m}(\sigma) \leq\|\varphi \mathbf{e}\|_{\infty} \omega_{d-1} M_{1}(\mathcal{D}, \nu, F) \rightarrow 0 \quad \text { as } \operatorname{size}(\mathcal{D}) \rightarrow 0 .
$$

Gathering these convergences in (3.6) and recalling that $\overline{\mathbf{v}}$ and $\bar{u}$ have been extended by 0 outside $\Omega$, we obtain

$$
\int_{\mathbb{R}^{d}} \overline{\mathbf{v}}(x) \cdot \varphi(x) \mathbf{e} \mathrm{d} x=-\int_{\mathbb{R}^{d}} \bar{u}(x) \operatorname{div}(\varphi \mathbf{e})(x) \mathrm{d} x,
$$

which concludes the proof.

\section{Proofs of Theorems 2.1 And 2.2}

Let us give an overview on the techniques used to prove the main theorems of this paper.

The first idea is, as usual, to obtain a priori estimates on the solution to the scheme. In order to do so, the basic technique (as already done in [12]) consists in multiplying each edge equation (2.2) by the fluxes; summing on the edges and gathering by control volumes thanks to (2.3) (which comes down to making a discrete integrate by parts), the right-hand side of (2.4) and the left-hand side of (2.5) naturally appear, which immediately leads to the desired estimates. These manipulations (multiplying the edges equations by some fluxes, summing and gathering by control volumes, identifying a reconstruction of gradient or a balance of fluxes) are recurrent in the handling of this mixed finite volume scheme and were used in the preceding section; rather than gathering them in abstract and general lemmas (which would anyhow need to be fitted to each situation), we prefer to repeat them when necessary, in order for the reader to become familiar with the techniques associated to our scheme.

Once a priori estimates are known, the compactness property of $L_{p, \nu}(\mathcal{D})$ gives a subsequence of the solution which weakly converges as $\operatorname{size}(\mathcal{D}) \rightarrow 0$. Since the problem is nonlinear, we cannot simply pass to the limit and we therefore come back to the monotony method of Minty-Browder, as used in the paper [22] of Leray-Lions. The trick is to write, for all regular $\varphi$, the discrete version of

$$
\int_{\Omega}(a(x, \nabla \varphi(x))-a(x, \mathbf{v}(x))) \cdot(\nabla \varphi(x)-\mathbf{v}(x)) \mathrm{d} x \geq 0
$$

(with, instead of $\nabla \varphi$, the function equal to the mean value of $\nabla \varphi$ on each control volume) and, using the basic manipulations described above, to pass to the limit in a similar way as in [22], thus proving that the weak limit $\bar{u}$ of the approximate solution is the weak solution to (1.1).

To conclude and prove the strong convergence of $\mathbf{v}$ to $\nabla \bar{u}$, one needs to use the exact solution $\bar{u}$ instead of $\varphi$ in the discrete counterpart of (4.7). However, in order to control the ensuing terms, because of (2.2) one must bound the error between $\bar{u}\left(\mathbf{x}_{L}\right)-\bar{u}\left(\mathbf{x}_{K}\right)$ and $\nabla \bar{u}\left(\mathbf{x}_{K}\right) \cdot\left(\mathbf{x}_{\sigma}-\mathbf{x}_{K}\right)+\nabla \bar{u}\left(\mathbf{x}_{L}\right) \cdot\left(\mathbf{x}_{L}-\mathbf{x}_{\sigma}\right)$ (or similar terms with the mean values of the functions on the control volumes instead of their values at $\mathbf{x}_{K}$ and $\left.\mathbf{x}_{L}\right)$; since $\bar{u}$ lacks regularity, such a bound does not exist in general. To overcome this difficulty, we use in (4.7) any regular function $\varphi$ which is close enough to $\bar{u}$, and we are then able to prove that the left-hand side of (4.7) with $\bar{u}$ instead of $\varphi$ is small if $\operatorname{size}(\mathcal{D})$ is small. This gives the a.e. convergence of $\mathbf{v}$ to $\nabla \bar{u}$, and the convergence in $L^{p}(\Omega)^{d}$ is then easy to obtain by classical techniques of monotone elliptic equations.

We now turn to the proof of the existence and uniqueness of a solution to the finite volume approximation. 
Proof of Theorem 2.1.

\section{Step 1. Existence.}

The proof is made by means of the topological degree.

Since $\xi \rightarrow a_{K}(\xi)$ is continuous (this comes from (1.2), (1.5) and the dominated convergence theorem), the non-linear system $((2.2)-(2.5))$ can be written as $\mathcal{G}(u, \mathbf{v}, F)=0$ with $\mathcal{G}$ continuous $H_{\mathcal{D}} \times H_{\mathcal{D}}^{d} \times \mathcal{F}_{\text {cons }} \rightarrow$ $H_{\mathcal{D}} \times H_{\mathcal{D}}^{d} \times \mathcal{F}_{\text {cons }}\left(\right.$ we define $\mathcal{F}_{\text {cons }}$ as the vector space of families $\left(F_{K, \sigma}\right)_{K \in \mathcal{M}, \sigma \in \mathcal{E}_{K}}$ such that $F_{K, \sigma}+F_{L, \sigma}=0$ whenever $\sigma=K \mid L \in \mathcal{E}_{\text {int }}$ ). The components of $\mathcal{G}$ are respectively given by the difference between the left-hand sides and right-hand sides of (2.5), (2.4) and (2.2).

For $t \in[0,1]$, let $\mathcal{G}_{t}$ be the application $H_{\mathcal{D}} \times H_{\mathcal{D}}^{d} \times \mathcal{F}_{\text {cons }} \rightarrow H_{\mathcal{D}} \times H_{\mathcal{D}}^{d} \times \mathcal{F}_{\text {cons }}$ defined, as $\mathcal{G}_{\text {, by }}(2.5),(2.4)$ and $(2.2)$ in which we have replaced $a_{K}(\xi)$ by $t a_{K}(\xi)+(1-t) \xi$ and $\left|F_{K, \sigma}\right|^{\frac{1}{p-1}-1} F_{K, \sigma}$ by $t\left|F_{K, \sigma}\right|^{\frac{1}{p-1}-1} F_{K, \sigma}+$ $(1-t) F_{K, \sigma}$. The function $t \rightarrow \mathcal{G}_{t}$ is a continuous homotopy between $\mathcal{G}_{0}$ and $\mathcal{G}_{1}=\mathcal{G}$. But $\mathcal{G}_{0}$ is an affine function which corresponds to an invertible system, by the results in [12] (the estimate we make below also shows that $\mathcal{G}_{0}$ is invertible); hence, for all $R$ large enough, denoting $B_{R}$ the ball of radius $R$ in $H_{\mathcal{D}} \times H_{\mathcal{D}}^{d} \times \mathcal{F}_{\text {cons }}$, we have $\operatorname{deg}\left(\mathcal{G}_{0}, B_{R}, 0\right) \neq 0$. If we manage to prove that, for $R$ large enough and for all $t \in[0,1]$, any solution to $\mathcal{G}_{t}(u, \mathbf{v}, F)=0$ satisfies $\|(u, \mathbf{v}, F)\|<R$, then the properties of the topological degree (see [10]) ensure that $\operatorname{deg}\left(\mathcal{G}, B_{R}, 0\right)=\operatorname{deg}\left(\mathcal{G}_{0}, B_{R}, 0\right) \neq 0$, and thus that there exists a solution in $B_{R}$ to $\mathcal{G}(u, \mathbf{v}, F)=0$.

Let $t \in[0,1]$ and $(u, \mathbf{v}, F)$ satisfy $\mathcal{G}_{t}(u, \mathbf{v}, F)=0$, that is to say

$$
\begin{gathered}
\mathbf{v}_{K} \cdot\left(\mathbf{x}_{\sigma}-\mathbf{x}_{K}\right)+\mathbf{v}_{L} \cdot\left(\mathbf{x}_{L}-\mathbf{x}_{\sigma}\right)+\nu_{K} \mathrm{~m}(K)\left[t\left|F_{K, \sigma}\right|^{\frac{1}{p-1}-1} F_{K, \sigma}+(1-t) F_{K, \sigma}\right] \\
-\nu_{L} \mathrm{~m}(L)\left[t\left|F_{L, \sigma}\right|^{\frac{1}{p-1}-1} F_{L, \sigma}+(1-t) F_{L, \sigma}\right]=u_{L}-u_{K}, \quad \forall \sigma=K \mid L \in \mathcal{E}_{\text {int }}, \\
\mathbf{v}_{K} \cdot\left(\mathbf{x}_{\sigma}-\mathbf{x}_{K}\right)+\nu_{K} \mathrm{~m}(K)\left[t\left|F_{K, \sigma}\right|^{\frac{1}{p-1}-1} F_{K, \sigma}+(1-t) F_{K, \sigma}\right]=-u_{K}, \\
\forall K \in \mathcal{M}, \forall \sigma \in \mathcal{E}_{K} \cap \mathcal{E}_{\text {ext }}, \\
F_{K, \sigma}+F_{L, \sigma}=0, \quad \forall \sigma=K \mid L \in \mathcal{E}_{\text {int }}, \\
\mathrm{m}(K)\left[t a_{K}\left(\mathbf{v}_{K}\right)+(1-t) \mathbf{v}_{K}\right]=\sum_{\sigma \in \mathcal{E}_{K}} F_{K, \sigma}\left(\mathbf{x}_{\sigma}-\mathbf{x}_{K}\right), \quad \forall K \in \mathcal{M}, \\
-\sum_{\sigma \in \mathcal{E}_{K}} F_{K, \sigma}=\int_{K} f(x) \mathrm{d} x, \quad \forall K \in \mathcal{M} .
\end{gathered}
$$

Multiply (4.8) by $F_{K, \sigma}$, sum over the edges and gather by control volumes using (4.9) and (4.11):

$$
\begin{aligned}
\sum_{K \in \mathcal{M}} \mathbf{v}_{K} \cdot \sum_{\sigma \in \mathcal{E}_{K}} F_{K, \sigma}\left(\mathbf{x}_{\sigma}-\mathbf{x}_{K}\right)+\sum_{K \in \mathcal{M}} \sum_{\sigma \in \mathcal{E}_{K}} \nu_{K} \operatorname{m}(K)\left[t\left|F_{K, \sigma}\right|^{\frac{p}{p-1}}+(1-t)\left|F_{K, \sigma}\right|^{2}\right] \\
=-\sum_{K \in \mathcal{M}} u_{K} \sum_{\sigma \in \mathcal{E}_{K}} F_{K, \sigma} \\
=\sum_{K \in \mathcal{M}} u_{K} f_{K}
\end{aligned}
$$

where $f_{K}=\int_{K} f(x) \mathrm{d} x$. By (4.10) and (1.3) we deduce, denoting $\mu_{\mathcal{D}}=\inf \left\{\nu_{K}, K \in \mathcal{M}\right\}>0$ and $\lambda_{\mathcal{D}}=$ $\inf \{\mathrm{m}(K), K \in \mathcal{M}\}>0$,

$$
\lambda_{\mathcal{D}}\left(t \alpha_{0}\left\|\left(\mathbf{v}_{K}\right)\right\|_{l^{p}}^{p}+(1-t)\left\|\left(\mathbf{v}_{K}\right)\right\|_{l^{2}}^{2}\right)+\mu_{\mathcal{D}} \lambda_{\mathcal{D}}\left(t \mid\left\|\left(F_{K, \sigma}\right)\right\|_{l^{p^{\prime}}}^{p^{\prime}}+(1-t)\left\|\left(F_{K, \sigma}\right)\right\|_{l^{2}}^{2}\right) \leq C_{7}\left\|\left(u_{K}\right)\right\|_{l^{1}}
$$


where $C_{7}$ does not depend on $t$ or $(u, \mathbf{v}, F)$ (we have denoted, for a finite family $\left(z_{i}\right)_{i \in I}$ and $r \in[1, \infty[$, $\left.\left\|\left(z_{i}\right)\right\|_{l^{r}}^{r}=\sum_{i \in I}\left|z_{i}\right|^{r}\right)$. Since all the norms on a finite dimensional space are equivalent, we deduce

$$
\left(t\left\|\left(\mathbf{v}_{K}\right)\right\|_{l^{1}}^{p}+(1-t)\left\|\left(\mathbf{v}_{K}\right)\right\|_{l^{1}}^{2}\right)+\left(t\left\|\left(F_{K, \sigma}\right)\right\|_{l^{1}}^{p^{\prime}}+(1-t)\left\|\left(F_{K, \sigma}\right)\right\|_{l^{1}}^{2}\right) \leq C_{8}\left\|\left(u_{K}\right)\right\|_{l^{1}}
$$

where $C_{8}$ depends on $\mathcal{D}$ but not on $t$ or $(u, \mathbf{v}, F)$.

Defining $\widetilde{F}_{K, \sigma}=t\left|F_{K, \sigma}\right|^{\frac{1}{p-1}-1} F_{K, \sigma}+(1-t) F_{K, \sigma}$, we notice that $(u, \mathbf{v}, \widetilde{F}) \in L_{2, \nu}(\mathcal{D})$; we can therefore apply Lemma 3.1 (with $p=2$ ) and use the fact that all the norms on a finite dimensional space are equivalent to find $C_{9}$ and $C_{10}$ depending on the discretization $\mathcal{D}$ but not on $t$ or $(u, \mathbf{v}, F)$ such that

$$
\begin{aligned}
\left\|\left(u_{K}\right)\right\|_{l^{1}} & \leq C_{9}\left(\left\|\left(\mathbf{v}_{K}\right)\right\|_{l^{1}}+\left\|\left(\widetilde{F}_{K, \sigma}\right)\right\|_{l^{1}}\right) \\
& \leq C_{9}\left(\left\|\left(\mathbf{v}_{K}\right)\right\|_{l^{1}}+t\left\|\left(\left|F_{K, \sigma}\right|^{\frac{1}{p-1}}\right)\right\|_{l^{1}}+(1-t)\left\|\left(F_{K, \sigma}\right)\right\|_{l^{1}}\right) \\
& \leq C_{10}\left(\left\|\left(\mathbf{v}_{K}\right)\right\|_{l^{1}}+t\left\|\left(F_{K, \sigma}\right)\right\|_{l^{1}}^{\frac{1}{p-1}}+\left\|\left(F_{K, \sigma}\right)\right\|_{l^{1}}\right)
\end{aligned}
$$

(we have used the inequality $\sum_{i \in I}\left|z_{i}\right|^{\frac{1}{p-1}} \leq \operatorname{Card}(I)\left(\sum_{i \in I}\left|z_{i}\right|\right)^{\frac{1}{p-1}}$, which is true since each $\left|z_{j}\right|$ is bounded by $\left.\sum_{i \in I}\left|z_{i}\right|\right)$. Injecting this in (4.13), we obtain

$$
\begin{aligned}
\left(t\left\|\left(\mathbf{v}_{K}\right)\right\|_{l^{1}}^{p}+(1-t)\left\|\left(\mathbf{v}_{K}\right)\right\|_{l^{1}}^{2}\right)+ & \left(t\left(\left\|\left(F_{K, \sigma}\right)\right\|_{l^{1}}^{\frac{1}{p-1}}\right)^{p}+(1-t)\left\|\left(F_{K, \sigma}\right)\right\|_{l^{1}}^{2}\right) \\
& \leq C_{11}\left(\left\|\left(\mathbf{v}_{K}\right)\right\|_{l^{1}}+t\left\|\left(F_{K, \sigma}\right)\right\|_{l^{1}}^{\frac{1}{p-1}}+\left\|\left(F_{K, \sigma}\right)\right\|_{l^{1}}\right)
\end{aligned}
$$

where $C_{11}$ does not depend on $t$ or $(u, \mathbf{v}, F)$.

For $t \geq \frac{1}{2}$, Young inequalities allow to deduce that

$$
\begin{aligned}
\frac{1}{2}\left\|\left(\mathbf{v}_{K}\right)\right\|_{l^{1}}^{p}+\frac{1}{2}\left(\left\|\left(F_{K, \sigma}\right)\right\|_{l^{1}}^{\frac{1}{p-1}}\right)^{p} & \leq C_{11}\left(\left\|\left(\mathbf{v}_{K}\right)\right\|_{l^{1}}+\left\|\left(F_{K, \sigma}\right)\right\|_{l^{1}}^{\frac{1}{p-1}}+\left\|\left(F_{K, \sigma}\right)\right\|_{l^{1}}\right) \\
& \leq C_{12}+\frac{1}{4}\left\|\left(\mathbf{v}_{K}\right)\right\|_{l^{1}}^{p}+\frac{1}{8}\left(\left\|\left(F_{K, \sigma}\right)\right\|_{l^{1}}^{\frac{1}{p-1}}\right)^{p}+\frac{1}{8}\left\|\left(F_{K, \sigma}\right)\right\|_{l^{1}}^{p^{1}}
\end{aligned}
$$

(where $C_{12}$ only depends on $p$ and $\left.C_{11}\right)$ and thus that $\left\|\left(\mathbf{v}_{K}\right)\right\|_{l^{1}}^{p}+\left\|\left(F_{K, \sigma}\right)\right\|_{l^{1}}^{p^{\prime}} \leq 4 C_{12}$.

For $t \leq \frac{1}{2}$, we write from (4.15):

$$
\begin{aligned}
\frac{1}{2}\left\|\left(\mathbf{v}_{K}\right)\right\|_{l^{1}}^{2}+t\left(\left\|\left(F_{K, \sigma}\right)\right\|_{l^{1}}^{\frac{1}{p-1}}\right)^{p}+\frac{1}{2}\left\|\left(F_{K, \sigma}\right)\right\|_{l^{1}}^{2} & \leq C_{11}\left(\left\|\left(\mathbf{v}_{K}\right)\right\|_{l^{1}}+t\left\|\left(F_{K, \sigma}\right)\right\|_{l^{1}}^{\frac{1}{p-1}}+\left\|\left(F_{K, \sigma}\right)\right\|_{l^{1}}\right) \\
& \leq C_{13}+\frac{1}{4}\left\|\left(\mathbf{v}_{K}\right)\right\|_{l^{1}}^{2}+\left(t\left\|\left(F_{K, \sigma}\right)\right\|_{l^{1}}^{\frac{1}{p-1}}\right)^{p}+\frac{1}{4}\left\|\left(F_{K, \sigma}\right)\right\|_{l^{1}}^{2} \\
& \leq C_{13}+\frac{1}{4}\left\|\left(\mathbf{v}_{K}\right)\right\|_{l^{1}}^{2}+t\left(\left\|\left(F_{K, \sigma}\right)\right\|_{l^{1}}^{\frac{1}{p-1}}\right)^{p}+\frac{1}{4}\left\|\left(F_{K, \sigma}\right)\right\|_{l^{1}}^{2}
\end{aligned}
$$

where $C_{13}$ only depends on $C_{11}$ and $p$ (we have used $t^{p-1} \leq 1$ ). Hence, here again we have an estimate: $\left\|\left(\mathbf{v}_{K}\right)\right\|_{l^{1}}^{2}+\left\|\left(F_{K, \sigma}\right)\right\|_{l^{1}}^{2} \leq 4 C_{13}$.

In either case, we find $R_{1}>0$ not depending on $t \in[0,1]$ or $(u, \mathbf{v}, F)$ such that $\left\|\left(\mathbf{v}_{K}\right)\right\|_{l^{1}}+\left\|\left(F_{K, \sigma}\right)\right\|_{l^{1}} \leq R_{1}$. Using this estimate in (4.14), we also deduce a bound on $\left\|\left(u_{K}\right)\right\|_{l^{1}}$, which concludes the proof of existence. 
Step 2. Uniqueness.

Assume that $(u, \mathbf{v}, F)$ and $\left(u^{\prime}, \mathbf{v}^{\prime}, F^{\prime}\right)$ are two solutions to $((2.2)-(2.5))$. Then, by (1.4),

$$
\begin{aligned}
0 \leq & \sum_{K \in \mathcal{M}} \mathrm{m}(K)\left(a_{K}\left(\mathbf{v}_{K}\right)-a_{K}\left(\mathbf{v}_{K}^{\prime}\right)\right) \cdot\left(\mathbf{v}_{K}-\mathbf{v}_{K}^{\prime}\right) \\
= & \sum_{K \in \mathcal{M}} \sum_{\sigma \in \mathcal{E}_{K}}\left(F_{K, \sigma}-F_{K, \sigma}^{\prime}\right)\left(\mathbf{x}_{\sigma}-\mathbf{x}_{K}\right) \cdot\left(\mathbf{v}_{K}-\mathbf{v}_{K}^{\prime}\right) \\
= & \sum_{\sigma \in \mathcal{E}}\left(F_{K, \sigma}-F_{K, \sigma}^{\prime}\right)\left[\left(\mathbf{v}_{K}-\mathbf{v}_{K}^{\prime}\right) \cdot\left(\mathbf{x}_{\sigma}-\mathbf{x}_{K}\right)+\left(\mathbf{v}_{L}-\mathbf{v}_{L}^{\prime}\right) \cdot\left(\mathbf{x}_{L}-\mathbf{x}_{\sigma}\right)\right] \\
= & \sum_{\sigma \in \mathcal{E}}\left(F_{K, \sigma}-F_{K, \sigma}^{\prime}\right)\left[\left(u_{L}-u_{L}^{\prime}\right)-\left(u_{K}-u_{K}^{\prime}\right)\right] \\
& -\sum_{\sigma \in \mathcal{E}}\left(F_{K, \sigma}-F_{K, \sigma}^{\prime}\right)\left[\nu_{K} \mathrm{~m}(K)\left|F_{K, \sigma}\right|^{r-1} F_{K, \sigma}-\nu_{L} \mathrm{~m}(L)\left|F_{L, \sigma}\right|^{r-1} F_{L, \sigma}\right. \\
& \left.-\left(\nu_{K} \mathrm{~m}(K)\left|F_{K, \sigma}^{\prime}\right|^{r-1} F_{K, \sigma}^{\prime}-\nu_{L} \mathrm{~m}(L)\left|F_{L, \sigma}^{\prime}\right|^{r-1} F_{L, \sigma}^{\prime}\right)\right]
\end{aligned}
$$

where $r=\frac{1}{p-1}$ and the quantities with index $L$ are defined as null if $\sigma \in \mathcal{E}_{\text {ext }} \cap \mathcal{E}_{K}$. Gathering these last sums by control volumes, we obtain

$$
\begin{aligned}
0 \leq & \sum_{K \in \mathcal{M}} \mathrm{m}(K)\left(a_{K}\left(\mathbf{v}_{K}\right)-a_{K}\left(\mathbf{v}_{K}^{\prime}\right)\right) \cdot\left(\mathbf{v}_{K}-\mathbf{v}_{K}^{\prime}\right) \\
= & -\sum_{K \in \mathcal{M}}\left(u_{K}-u_{K}^{\prime}\right) \sum_{\sigma \in \mathcal{E}_{K}}\left(F_{K, \sigma}-F_{K, \sigma}^{\prime}\right) \\
& -\sum_{K \in \mathcal{M}} \sum_{\sigma \in \mathcal{E}_{K}} \nu_{K} \mathrm{~m}(K)\left(F_{K, \sigma}-F_{K, \sigma}^{\prime}\right)\left[\left|F_{K, \sigma}\right|^{r-1} F_{K, \sigma}-\left|F_{K, \sigma}^{\prime}\right|^{r-1} F_{K, \sigma}^{\prime}\right] \\
= & -\sum_{K \in \mathcal{M}} \sum_{\sigma \in \mathcal{E}_{K}} \nu_{K} \mathrm{~m}(K)\left(F_{K, \sigma}-F_{K, \sigma}^{\prime}\right)\left[\left|F_{K, \sigma}\right|^{r-1} F_{K, \sigma}-\left|F_{K, \sigma}^{\prime}\right|^{r-1} F_{K, \sigma}^{\prime}\right]
\end{aligned}
$$

since $\sum_{\sigma \in \mathcal{E}_{K}} F_{K, \sigma}-\sum_{\sigma \in \mathcal{E}_{K}} F_{K, \sigma}^{\prime}=-\int_{K} f(x) \mathrm{d} x+\int_{K} f(x) \mathrm{d} x=0$. As $X \rightarrow|X|^{r-1} X$ is non-decreasing on $\mathbb{R}$, we deduce

$$
\begin{aligned}
0 & \leq \sum_{K \in \mathcal{M}} \mathrm{m}(K)\left(a_{K}\left(\mathbf{v}_{K}\right)-a_{K}\left(\mathbf{v}_{K}^{\prime}\right)\right) \cdot\left(\mathbf{v}_{K}-\mathbf{v}_{K}^{\prime}\right) \\
& =-\sum_{K \in \mathcal{M}} \sum_{\sigma \in \mathcal{E}_{K}} \nu_{K} \mathrm{~m}(K)\left(F_{K, \sigma}-F_{K, \sigma}^{\prime}\right)\left[\left|F_{K, \sigma}\right|^{r-1} F_{K, \sigma}-\left|F_{K, \sigma}^{\prime}\right|^{r-1} F_{K, \sigma}^{\prime}\right] \\
& \leq 0
\end{aligned}
$$

and thus all the terms (which are non-negative) in these sums are null. By (1.4), this implies $\mathbf{v}_{K}=\mathbf{v}_{K}^{\prime}$ for all $K \in \mathcal{M}$ and, since $X \rightarrow|X|^{r-1} X$ is one-to-one, $F_{K, \sigma}=F_{K, \sigma}^{\prime}$ for all $K \in \mathcal{M}$ and all $\sigma \in \mathcal{E}_{K}$. We then use the equations (2.2) linking $u$ to $(\mathbf{v}, F)$ and $u^{\prime}$ to $\left(\mathbf{v}^{\prime}, F^{\prime}\right)$ to conclude that $u_{K}=u_{K}^{\prime}$ for all $K \in \mathcal{M}$ (first for the control volumes on the boundary of $\Omega$ and then, successively, for all the control volumes inside $\Omega$ ).

Before proving Theorem 2.2, we need more precise estimates on the solution to the finite volume approximation. 
Lemma 4.1 (estimate on the discrete solution). Assume Hypotheses (1.2)-(1.6). Let $\mathcal{D}$ be an admissible discretization of $\Omega$ such that $\operatorname{regul}(\mathcal{D}) \leq \theta$ for some $\theta>0$. Let $\left(\nu_{K}\right)_{K \in \mathcal{M}}$ be a family of positive real numbers such that, for some $\nu_{0}>0$ and some $\beta \geq-p^{\prime}(d-1), \nu_{K} \leq \nu_{0} \operatorname{diam}(K)^{\beta}$ for all $K \in \mathcal{M}$. Let $(u, \mathbf{v}, F)$ be the solution to $((2.2)-(2.5))$. Then there exists $C_{14}$ only depending on $d, p, \Omega, \theta, f, \nu_{0}, \beta$ and $\alpha_{0}$ such that

$$
\|\mathbf{v}\|_{L^{p}(\Omega)^{d}}^{p}+\sum_{K \in \mathcal{M}} \sum_{\sigma \in \mathcal{E}_{K}} \nu_{K}\left(\left|F_{K, \sigma}\right|^{\frac{1}{p-1}}\right)^{p} \mathrm{~m}(K) \leq C_{14} .
$$

Proof of Lemma 4.1. Multiply (2.2) by $F_{K, \sigma}$, sum over the edges and gather by control volumes using (2.3). This leads to

$$
\sum_{K \in \mathcal{M}} \mathbf{v}_{K} \cdot \sum_{\sigma \in \mathcal{E}_{K}} F_{K, \sigma}\left(\mathbf{x}_{\sigma}-\mathbf{x}_{K}\right)+\sum_{K \in \mathcal{M}} \sum_{\sigma \in \mathcal{E}_{K}} \nu_{K} \mathrm{~m}(K)\left|F_{K, \sigma}\right|^{\frac{1}{p-1}+1}=-\sum_{K \in \mathcal{M}} u_{K} \sum_{\sigma \in \mathcal{E}_{K}} F_{K, \sigma} .
$$

By (2.4) and (2.5), we deduce

$$
\sum_{K \in \mathcal{M}} \mathrm{m}(K) a_{K}\left(\mathbf{v}_{K}\right) \cdot \mathbf{v}_{K}+\sum_{K \in \mathcal{M}} \sum_{\sigma \in \mathcal{E}_{K}} \nu_{K} \mathrm{~m}(K)\left(\left|F_{K, \sigma}\right|^{\frac{1}{p-1}}\right)^{p}=\int_{\Omega} f(x) u(x) \mathrm{d} x
$$

and, thanks to Hypothesis (1.3) and Young inequality, for all $\varepsilon>0$,

$$
\begin{aligned}
\alpha_{0}\|\mathbf{v}\|_{L^{p}(\Omega)^{d}}^{p}+\sum_{K \in \mathcal{M}} \sum_{\sigma \in \mathcal{E}_{K}} \nu_{K} \operatorname{m}(K)\left(\left|F_{K, \sigma}\right|^{\frac{1}{p-1}}\right)^{p} & \leq\|f\|_{L^{p^{\prime}}(\Omega)}\|u\|_{L^{p}(\Omega)} \\
& \leq \frac{1}{p^{\prime}(p \varepsilon)^{\frac{p^{\prime}}{p}}}\|f\|_{L^{p^{\prime}(\Omega)}}^{p^{\prime}}+\varepsilon\|u\|_{L^{p}(\Omega)}^{p} .
\end{aligned}
$$

We now apply Lemma 3.1 to find $C_{15}$ only depending on $d, p, \Omega, \theta$ and $f$ such that

$$
\begin{aligned}
& \alpha_{0}\|\mathbf{v}\|_{L^{p}(\Omega)^{d}}^{p}+\sum_{K \in \mathcal{M}} \sum_{\sigma \in \mathcal{E}_{K}} \nu_{K}\left(\left|F_{K, \sigma}\right|^{\frac{1}{p-1}}\right)^{p} \mathrm{~m}(K) \\
& \leq C_{15} \varepsilon^{-\frac{p^{\prime}}{p}}+\varepsilon C_{15}\|\mathbf{v}\|_{L^{p}(\Omega)^{d}}^{p}+\varepsilon C_{15} \sum_{K \in \mathcal{M}} \sum_{\sigma \in \mathcal{E}_{K}} \operatorname{diam}(K)^{(d-1) p} \nu_{K}^{p}\left(\left|F_{K, \sigma}\right|^{\frac{1}{p-1}}\right)^{p} \mathrm{~m}(K) \\
& \leq C_{15} \varepsilon^{-\frac{p^{\prime}}{p}}+\varepsilon C_{15}\|\mathbf{v}\|_{L^{p}(\Omega)^{d}}^{p}+\varepsilon C_{15} \sum_{K \in \mathcal{M}} \sum_{\sigma \in \mathcal{E}_{K}}\left(\operatorname{diam}(K)^{(d-1) p} \nu_{K}^{p-1}\right) \nu_{K}\left(\left|F_{K, \sigma}\right|^{\frac{1}{p-1}}\right)^{p} \mathrm{~m}(K) .
\end{aligned}
$$

Since $\nu_{K} \leq \nu_{0} \operatorname{diam}(K)^{\beta}$, we have

$$
\operatorname{diam}(K)^{(d-1) p} \nu_{K}^{p-1} \leq \nu_{0}^{p-1} \operatorname{diam}(K)^{(d-1) p+\beta(p-1)} \leq \nu_{0}^{p-1} \operatorname{diam}(\Omega)^{(d-1) p+\beta(p-1)}
$$

(we have used $(d-1) p+\beta(p-1) \geq 0$, which is true since $\left.(d-1) p^{\prime}+\beta \geq 0\right)$. Hence, there exists $C_{16}$ only depending on $d, p, \Omega, \theta, f, \nu_{0}$ and $\beta$ such that

$$
\begin{aligned}
\alpha_{0}\|\mathbf{v}\|_{L^{p}(\Omega)^{d}}^{p} & +\sum_{K \in \mathcal{M}} \sum_{\sigma \in \mathcal{E}_{K}} \nu_{K}\left(\left|F_{K, \sigma}\right|^{\frac{1}{p-1}}\right)^{p} \mathrm{~m}(K) \\
& \leq C_{15} \varepsilon^{-\frac{p^{\prime}}{p}}+\varepsilon C_{15}\|\mathbf{v}\|_{L^{p}(\Omega)^{d}}^{p}+\varepsilon C_{16} \sum_{K \in \mathcal{M}} \sum_{\sigma \in \mathcal{E}_{K}} \nu_{K}\left(\left|F_{K, \sigma}\right|^{\frac{1}{p-1}}\right)^{p} \mathrm{~m}(K)
\end{aligned}
$$

and the choice $\varepsilon=\inf \left(\frac{\alpha_{0}}{2 C_{15}}, \frac{1}{2 C_{16}}\right)$ concludes the proof. 
Let us now prove the convergence of the finite volume approximation.

Proof of Theorem 2.2.

Step 1. Convergence of $(u, \mathbf{v})$.

For simplicity, we drop the indices $n$. We have

$$
\begin{aligned}
M_{p}(\mathcal{D}, \nu, F)^{p} & =\sum_{K \in \mathcal{M}} \sum_{\sigma \in \mathcal{E}_{K}} \operatorname{diam}(K)^{(d-1) p} \nu_{K}^{p-1} \nu_{K}\left(\left|F_{K, \sigma}\right|^{\frac{1}{p-1}}\right)^{p} \mathrm{~m}(K) \\
& =\nu_{0}^{p-1} \sum_{K \in \mathcal{M}} \sum_{\sigma \in \mathcal{E}_{K}} \operatorname{diam}(K)^{(d-1) p+\beta(p-1)} \nu_{K}\left(\left|F_{K, \sigma}\right|^{\frac{1}{p-1}}\right)^{p} \mathrm{~m}(K) \\
& \leq \nu_{0}^{p-1} \operatorname{size}(\mathcal{D})^{(d-1) p+\beta(p-1)} \sum_{K \in \mathcal{M}} \sum_{\sigma \in \mathcal{E}_{K}} \nu_{K}\left(\left|F_{K, \sigma}\right|^{\frac{1}{p-1}}\right)^{p} \mathrm{~m}(K)
\end{aligned}
$$

since $(d-1) p+\beta(p-1)>0$ (because $\beta>-p^{\prime}(d-1)$ ). Hence, by the assumptions and Lemma $4.1, M_{p}(\mathcal{D}, \nu, F) \rightarrow$ 0 (and is thus bounded) as $\operatorname{size}(\mathcal{D}) \rightarrow 0$. Moreover,

$$
\begin{aligned}
M_{1}(\mathcal{D}, \nu, F) & =\sum_{K \in \mathcal{M}} \sum_{\sigma \in \mathcal{E}_{K}} \operatorname{diam}(K)^{d-1} \nu_{K}\left|F_{K, \sigma}\right|^{\frac{1}{p-1}} \mathrm{~m}(K) \\
& \leq\left(\sum_{K \in \mathcal{M}} \sum_{\sigma \in \mathcal{E}_{K}} \operatorname{diam}(K)^{(d-1) p} \nu_{K}^{p}\left(\left|F_{K, \sigma}\right|^{\frac{1}{p-1}}\right)^{p} \mathrm{~m}(K)\right)^{\frac{1}{p}}\left(\sum_{K \in \mathcal{M}} \sum_{\sigma \in \mathcal{E}_{K}} \mathrm{~m}(K)\right)^{\frac{1}{p^{\prime}}} \\
& \leq M_{p}(\mathcal{D}, \nu, F) \operatorname{regul}(\mathcal{D})^{\frac{1}{p^{\prime}}} \mathrm{m}(\Omega)^{\frac{1}{p^{\prime}}}
\end{aligned}
$$

and therefore $M_{1}(\mathcal{D}, \nu, F) \rightarrow 0$ as $\operatorname{size}(\mathcal{D}) \rightarrow 0$. Since $\mathbf{v}$ is bounded in $L^{p}(\Omega)^{d}$ (Lem. 4.1), the assumptions of Lemma 3.3 are fulfilled and there exists $\bar{u} \in W_{0}^{1, p}(\Omega)$ such that, up to a subsequence, as $\operatorname{size}(\mathcal{D}) \rightarrow 0, u \rightarrow \bar{u}$ weakly in $L^{p}(\Omega)$ and strongly in $L^{q}(\Omega)$ for all $q<p$, and $\mathbf{v} \rightarrow \nabla \bar{u}$ weakly in $L^{p}(\Omega)^{d}$. We now prove that $\bar{u}$ is a weak solution to (1.1); since this solution is unique, this will prove that the whole sequences $(u, \mathbf{v})$, not only subsequences, converge.

Step 2. The limit is a solution to (1.1).

To prove that $\bar{u}$ is a weak solution to $(1.1)$, since the problem is non-linear and since we only have the weak convergence of $\mathbf{v}$, we copy the monotony method of Minty-Browder used in [22].

Let $\varphi \in C_{c}^{\infty}(\Omega)$ and denote $[\nabla \varphi]_{K}$ the mean value of $\nabla \varphi$ on the control volume $K$. By the monotony hypothesis (1.4), we can write

$$
0 \leq \sum_{K \in \mathcal{M}} \mathrm{m}(K)\left(a_{K}\left([\nabla \varphi]_{K}\right)-a_{K}\left(\mathbf{v}_{K}\right)\right) \cdot\left([\nabla \varphi]_{K}-\mathbf{v}_{K}\right)
$$

We have

$$
\sum_{K \in \mathcal{M}} \mathrm{m}(K) a_{K}\left([\nabla \varphi]_{K}\right) \cdot\left([\nabla \varphi]_{K}-\mathbf{v}_{K}\right)=\int_{\Omega} a_{\mathcal{D}}\left(x,(\nabla \varphi)_{\mathcal{D}}(x)\right) \cdot\left((\nabla \varphi)_{\mathcal{D}}(x)-\mathbf{v}(x)\right) \mathrm{d} x
$$

where $a_{\mathcal{D}}(\cdot, \xi)$ is the piecewise constant function equal to $a_{K}(\xi)$ on each control volume $K$ and $(\nabla \varphi)_{\mathcal{D}}$ is the piecewise constant function equal to $[\nabla \varphi]_{K}$ on each control volume $K$. Lemma 8.3 shows that, as $\operatorname{size}(\mathcal{D}) \rightarrow 0$, $a_{\mathcal{D}}\left(\cdot,(\nabla \varphi)_{\mathcal{D}}\right) \rightarrow a(\cdot, \nabla \varphi)$ strongly in $L^{p^{\prime}}(\Omega)^{d}$, and it is quite clear that $(\nabla \varphi)_{\mathcal{D}} \rightarrow \nabla \varphi$ strongly in $L^{p}(\Omega)^{d}$. 
Since $\mathbf{v} \rightarrow \nabla \bar{u}$ weakly in $L^{p}(\Omega)^{d}$, we deduce that

$$
\sum_{K \in \mathcal{M}} \mathrm{m}(K) a_{K}\left([\nabla \varphi]_{K}\right) \cdot\left([\nabla \varphi]_{K}-\mathbf{v}_{K}\right) \rightarrow \int_{\Omega} a(x, \nabla \varphi(x)) \cdot(\nabla \varphi(x)-\nabla \bar{u}(x)) \mathrm{d} x \quad \text { as } \operatorname{size}(\mathcal{D}) \rightarrow 0 .
$$

By (2.4) and (2.3), we find

$$
\begin{aligned}
\sum_{K \in \mathcal{M}} \mathrm{m}(K) a_{K}\left(\mathbf{v}_{K}\right) \cdot[\nabla \varphi]_{K} & =\sum_{K \in \mathcal{M}} \sum_{\sigma \in \mathcal{E}_{K}} F_{K, \sigma}\left(\mathbf{x}_{\sigma}-\mathbf{x}_{K}\right) \cdot[\nabla \varphi]_{K} \\
& =\sum_{\sigma \in \mathcal{E}_{\text {int }}, \sigma=K \mid L} F_{K, \sigma}\left([\nabla \varphi]_{K} \cdot\left(\mathbf{x}_{\sigma}-\mathbf{x}_{K}\right)+[\nabla \varphi]_{L} \cdot\left(\mathbf{x}_{L}-\mathbf{x}_{\sigma}\right)\right)
\end{aligned}
$$

(we take $\operatorname{size}(\mathcal{D})$ small enough so that $\varphi=0$ on the control volumes which touch the boundary of $\Omega$ ). Since $\varphi$ is regular, we have

$$
[\nabla \varphi]_{K} \cdot\left(\mathbf{x}_{\sigma}-\mathbf{x}_{K}\right)+[\nabla \varphi]_{L} \cdot\left(\mathbf{x}_{L}-\mathbf{x}_{\sigma}\right)=\varphi\left(\mathbf{x}_{L}\right)-\varphi\left(\mathbf{x}_{K}\right)+R_{K, L}
$$

where $\left|R_{K, L}\right| \leq C_{17}\left(\operatorname{diam}(K)^{2}+\operatorname{diam}(L)^{2}\right)$ with $C_{17}$ only depending on $\varphi$. Hence, gathering by control volumes thanks to $(2.3)$, using $(2.5)$ and recalling that $\varphi\left(\mathbf{x}_{K}\right)=0$ if $\mathcal{E}_{K} \cap \mathcal{E}_{\text {ext }} \neq \emptyset$,

$$
\begin{aligned}
\sum_{K \in \mathcal{M}} \mathrm{m}(K) a_{K}\left(\mathbf{v}_{K}\right) \cdot[\nabla \varphi]_{K} & =\sum_{\sigma \in \mathcal{E}_{\text {int }}, \sigma=K \mid L} F_{K, \sigma}\left(\varphi\left(\mathbf{x}_{L}\right)-\varphi\left(\mathbf{x}_{K}\right)\right)+T_{7} \\
& =-\sum_{K \in \mathcal{M}} \varphi\left(\mathbf{x}_{K}\right) \sum_{\sigma \in \mathcal{E}_{K}} F_{K, \sigma}+T_{7} \\
& =\sum_{K \in \mathcal{M}} \varphi\left(\mathbf{x}_{K}\right) \int_{K} f(x) \mathrm{d} x+T_{7}
\end{aligned}
$$

where $\left|T_{7}\right| \leq C_{17} \sum_{K \in \mathcal{M}} \sum_{\sigma \in \mathcal{E}_{K}} \operatorname{diam}(K)^{2}\left|F_{K, \sigma}\right|$. We now write

$$
\begin{aligned}
\left|T_{7}\right| & \leq C_{17} \sum_{K \in \mathcal{M}} \sum_{\sigma \in \mathcal{E}_{K}}\left(\operatorname{diam}(K)^{2}\left(\nu_{K} \mathrm{~m}(K)\right)^{-\frac{1}{p^{\prime}}}\right) \times\left(\left|F_{K, \sigma}\right|\left(\nu_{K} \mathrm{~m}(K)\right)^{\frac{1}{p^{\prime}}}\right) \\
& \leq C_{17}\left(\sum_{K \in \mathcal{M}} \sum_{\sigma \in \mathcal{E}_{K}} \operatorname{diam}(K)^{2 p}\left(\nu_{K} \mathrm{~m}(K)\right)^{-\frac{p}{p^{\prime}}}\right)^{\frac{1}{p}}\left(\sum_{K \in \mathcal{M}} \sum_{\sigma \in \mathcal{E}_{K}}\left|F_{K, \sigma}\right|^{p^{\prime}} \nu_{K} \mathrm{~m}(K)\right)^{\frac{1}{p^{\prime}}}
\end{aligned}
$$

By Lemma 4.1, the second factor of this right-hand side is bounded. Moreover,

$$
\begin{aligned}
\sum_{K \in \mathcal{M}} \sum_{\sigma \in \mathcal{E}_{K}} \operatorname{diam}(K)^{2 p}\left(\nu_{K} \mathrm{~m}(K)\right)^{-\frac{p}{p^{\prime}}} & \leq \nu_{0}^{-\frac{p}{p^{\prime}}} \operatorname{regul}(\mathcal{D}) \sum_{K \in \mathcal{M}} \operatorname{diam}(K)^{2 p-\beta \frac{p}{p^{\prime}}} \mathrm{m}(K)^{-\frac{p}{p^{\prime}}-1} \mathrm{~m}(K) \\
& \leq \nu_{0}^{-\frac{p}{p^{\prime}}} \operatorname{regul}(\mathcal{D}) \sum_{K \in \mathcal{M}} \operatorname{diam}(K)^{2 p-\beta(p-1)} \mathrm{m}(K)^{-p} \mathrm{~m}(K)
\end{aligned}
$$

Using (2.1), and since $(2-d) p-\beta(p-1)>0$ (because $\beta<-p^{\prime}(d-2)$ ), we find

$$
\begin{aligned}
\sum_{K \in \mathcal{M}} \sum_{\sigma \in \mathcal{E}_{K}} \operatorname{diam}(K)^{2 p}\left(\nu_{K} \mathrm{~m}(K)\right)^{-\frac{p}{p^{\prime}}} & \leq \nu_{0}^{-\frac{p}{p^{\prime}}} \operatorname{regul}(\mathcal{D}) \frac{\operatorname{regul}(\mathcal{D})^{p}}{\omega_{d}^{p}} \sum_{K \in \mathcal{M}} \operatorname{diam}(K)^{2 p-\beta(p-1)-d p} \mathrm{~m}(K) \\
& \leq \nu_{0}^{-\frac{p}{p^{\prime}}} \operatorname{regul}(\mathcal{D}) \frac{\operatorname{regul}(\mathcal{D})^{p}}{\omega_{d}^{p}} \operatorname{size}(\mathcal{D})^{(2-d) p-\beta(p-1)} \mathrm{m}(\Omega) .
\end{aligned}
$$


This last term tends to 0 as $\operatorname{size}(\mathcal{D}) \rightarrow 0$ (recall that $\operatorname{regul}(\mathcal{D})$ is bounded), and we deduce from $(4.23)$ that $T_{7} \rightarrow 0$ as $\operatorname{size}(\mathcal{D}) \rightarrow 0$. Coming back to $(4.22)$, we obtain

$$
\sum_{K \in \mathcal{M}} \mathrm{m}(K) a_{K}\left(\mathbf{v}_{K}\right) \cdot[\nabla \varphi]_{K} \rightarrow \int_{\Omega} f(x) \varphi(x) \mathrm{d} x \quad \text { as } \operatorname{size}(\mathcal{D}) \rightarrow 0 .
$$

We have seen in the proof of Lemma 4.1 that $(u, \mathbf{v}, F)$, solution to $((2.2)-(2.5))$, satisfies $(4.16)$; in particular,

$$
\sum_{K \in \mathcal{M}} \mathrm{m}(K) a_{K}\left(\mathbf{v}_{K}\right) \cdot \mathbf{v}_{K} \leq \int_{\Omega} f(x) u(x) \mathrm{d} x \rightarrow \int_{\Omega} f(x) \bar{u}(x) \mathrm{d} x \quad \text { as } \operatorname{size}(\mathcal{D}) \rightarrow 0 .
$$

Thanks to $(4.20),(4.24)$ and (4.25), we can take the limsup of $(4.19)$ as $\operatorname{size}(\mathcal{D}) \rightarrow 0$ and we find

$$
\begin{aligned}
0 & \leq \int_{\Omega} a(x, \nabla \varphi(x)) \cdot(\nabla \varphi(x)-\nabla \bar{u}(x)) \mathrm{d} x-\int_{\Omega} f(x) \varphi(x) \mathrm{d} x+\int_{\Omega} f(x) \bar{u}(x) \mathrm{d} x \\
& =\int_{\Omega} a(x, \nabla \varphi(x)) \cdot(\nabla \varphi(x)-\nabla \bar{u}(x)) \mathrm{d} x-\int_{\Omega} f(x)(\varphi(x)-\bar{u}(x)) \mathrm{d} x .
\end{aligned}
$$

It is well known that such an inequality, valid for all regular $\varphi$, implies that $\bar{u}$ is a weak solution of (1.1); for the sake of completeness, we nevertheless make the proof.

Let $\psi \in C_{c}^{\infty}(\Omega), t>0$ and $\left(\varphi_{n}\right)_{n \geq 1}$ be functions in $C_{c}^{\infty}(\Omega)$ which converge in $W_{0}^{1, p}(\Omega)$ to $\bar{u}+t \psi$. We can apply (4.26) to $\varphi_{n}$ and pass to the limit $n \rightarrow \infty$, thanks to (1.2) and (1.5) (for example, we extract a subsequence such that $\nabla \varphi_{n} \rightarrow \nabla \bar{u}+t \nabla \psi$ a.e. and we use Vitali theorem); this gives

$$
\int_{\Omega} a(x, \nabla \bar{u}(x)+t \nabla \psi(x)) \cdot(t \nabla \psi(x)) \mathrm{d} x-\int_{\Omega} t f(x) \psi(x) \mathrm{d} x \geq 0 .
$$

Dividing this inequality by $t$ and letting $t \rightarrow 0^{+}$(still using (1.2) and (1.5)), we obtain $\int_{\Omega} a(x, \nabla \bar{u}(x)) \cdot \nabla \psi(x) \mathrm{d} x-$ $\int_{\Omega} f(x) \psi(x) \mathrm{d} x \geq 0$. Replacing $\psi$ by $-\psi$, we see that this inequality is in fact an equality, which proves that $\bar{u}$ is a weak solution to (1.1).

Step 3. Strong convergence of $\mathbf{v}$.

It remains to prove that $\mathbf{v} \rightarrow \nabla \bar{u}$ strongly in $L^{p}(\Omega)^{d}$. As usual, we only prove the strong convergence of a subsequence and we use the uniqueness of the limit to obtain the strong convergence of the whole sequence. The methods used here are quite similar to the classical techniques of non-linear elliptic equations; however, since we cannot directly put a discretization of $\bar{u}$ as a test function in our finite volume scheme (because $\bar{u}$ is not regular enough), we have to adapt these techniques by taking regular functions which approximate $\bar{u}$.

Let us first prove that, up to a subsequence, $\mathbf{v} \rightarrow \nabla \bar{u}$ almost everywhere on $\Omega$. Let $\varepsilon \in] 0,1[$ and take $\left(\varphi_{j}\right)_{j \geq 1} \in C_{c}^{\infty}(\Omega)$ such that $\varphi_{j} \rightarrow \bar{u}$ in $W_{0}^{1, p}(\Omega)$ as $j \rightarrow \infty$. We have

$$
\begin{aligned}
\int_{\Omega}(a(x, \nabla \bar{u}(x))-a(x, \mathbf{v}(x))) \cdot(\nabla \bar{u}(x)-\mathbf{v}(x)) \mathrm{d} x \leq & \left\|a(\cdot, \nabla \bar{u})-a\left(\cdot, \nabla \varphi_{j}\right)\right\|_{L^{p^{\prime}}(\Omega)^{d}}\left(\|\nabla \bar{u}\|_{L^{p}(\Omega)^{d}}+\|\mathbf{v}\|_{L^{p}(\Omega)^{d}}\right) \\
& +\int_{\Omega}\left(a\left(x, \nabla \varphi_{j}(x)\right)-a(x, \mathbf{v}(x))\right) \cdot(\nabla \bar{u}(x)-\mathbf{v}(x)) \mathrm{d} x \\
\leq & \left\|a(\cdot, \nabla \bar{u})-a\left(\cdot, \nabla \varphi_{j}\right)\right\|_{L^{p^{\prime}}(\Omega)^{d}}\left(\|\nabla \bar{u}\|_{L^{p}(\Omega)^{d}}+\|\mathbf{v}\|_{L^{p}(\Omega)^{d}}\right) \\
& +\left(\left\|a\left(\cdot, \nabla \varphi_{j}\right)\right\|_{L^{p^{\prime}}(\Omega)^{d}}+\|a(\cdot, \mathbf{v})\|_{L^{p^{\prime}}(\Omega)^{d}}\right)\left\|\nabla \bar{u}-\nabla \varphi_{j}\right\|_{L^{p}(\Omega)^{d}} \\
& +\int_{\Omega}\left(a\left(x, \nabla \varphi_{j}(x)\right)-a(x, \mathbf{v}(x))\right) \cdot\left(\nabla \varphi_{j}(x)-\mathbf{v}(x)\right) \mathrm{d} x .
\end{aligned}
$$


Since $\mathbf{v}$ and $\nabla \varphi_{j}$ are bounded in $L^{p}(\Omega)^{d}$, (1.5) shows that $a(\cdot, \mathbf{v})$ and $a\left(\cdot, \nabla \varphi_{j}\right)$ are bounded in $L^{p^{\prime}}(\Omega)^{d}$, independently of $j$ or $\mathcal{D}$. Moreover, since $\varphi_{j} \rightarrow \bar{u}$ in $W_{0}^{1, p}(\Omega),(1.2)$ and (1.5) allow to see that $a\left(\cdot, \nabla \varphi_{j}\right) \rightarrow$ $a(\cdot, \nabla \bar{u})$ in $L^{p^{\prime}}(\Omega)^{d}$ as $j \rightarrow \infty$. Hence, we can fix $j=J$ not depending on $\mathcal{D}$ such that $\left\|\varphi_{J}-\bar{u}\right\|_{W_{0}^{1, p}(\Omega)} \leq \varepsilon$ and

$$
\int_{\Omega}(a(x, \nabla \bar{u}(x))-a(x, \mathbf{v}(x))) \cdot(\nabla \bar{u}(x)-\mathbf{v}(x)) \mathrm{d} x \leq \varepsilon+\int_{\Omega}\left(a\left(x, \nabla \varphi_{J}(x)\right)-a(x, \mathbf{v}(x))\right) \cdot\left(\nabla \varphi_{J}(x)-\mathbf{v}(x)\right) \mathrm{d} x .
$$

We have $\left(\nabla \varphi_{J}\right)_{\mathcal{D}} \rightarrow \nabla \varphi_{J}$ in $L^{p}(\Omega)^{d}$ as $\operatorname{size}(\mathcal{D}) \rightarrow 0$, which implies $a\left(\cdot,\left(\nabla \varphi_{J}\right)_{\mathcal{D}}\right) \rightarrow a\left(\cdot, \nabla \varphi_{J}\right)$ in $L^{p^{\prime}}(\Omega)^{d}$. Using the fact that $\mathbf{v}$ is bounded in $L^{p}(\Omega)^{d}$, we deduce that, for $\operatorname{size}(\mathcal{D})$ small enough,

$$
\int_{\Omega}(a(x, \nabla \bar{u}(x))-a(x, \mathbf{v}(x))) \cdot(\nabla \bar{u}(x)-\mathbf{v}(x)) \mathrm{d} x \leq 2 \varepsilon+\int_{\Omega}\left(a\left(x,\left(\nabla \varphi_{J}\right)_{\mathcal{D}}(x)\right)-a(x, \mathbf{v}(x))\right) \cdot\left(\left(\nabla \varphi_{J}\right)_{\mathcal{D}}(x)-\mathbf{v}(x)\right) \mathrm{d} x .
$$

But

$\int_{\Omega}\left(a\left(x,\left(\nabla \varphi_{J}\right)_{\mathcal{D}}(x)\right)-a(x, \mathbf{v}(x))\right) \cdot\left(\left(\nabla \varphi_{J}\right)_{\mathcal{D}}(x)-\mathbf{v}(x)\right) \mathrm{d} x=\sum_{K \in \mathcal{M}} \mathrm{m}(K)\left(a_{K}\left(\left[\nabla \varphi_{J}\right]_{K}\right)-a_{K}\left(\mathbf{v}_{K}\right)\right) \cdot\left(\left[\nabla \varphi_{J}\right]_{K}-\mathbf{v}_{K}\right)$,

which is the right-hand side of (4.19) with $\varphi=\varphi_{J}$. The reasoning made in Step 2 (recall that $\varphi_{J}$ is regular) shows that this term is bounded from above by a quantity which tends, as $\operatorname{size}(\mathcal{D}) \rightarrow 0$, to $\int_{\Omega} a\left(x, \nabla \varphi_{J}(x)\right)$. $\left(\nabla \varphi_{J}(x)-\nabla \bar{u}(x)\right) \mathrm{d} x-\int_{\Omega} f(x)\left(\varphi_{J}(x)-\bar{u}(x)\right) \mathrm{d} x$. Since we have chosen $\varphi_{J}$ so that $\left\|\varphi_{J}-\bar{u}\right\|_{W_{0}^{1, p}(\Omega)} \leq \varepsilon,(1.5)$ implies

$$
\begin{aligned}
\mid \int_{\Omega} a\left(x, \nabla \varphi_{J}(x)\right) \cdot\left(\nabla \varphi_{J}(x)-\nabla \bar{u}(x)\right) \mathrm{d} x & -\int_{\Omega} f(x)\left(\varphi_{J}(x)-\bar{u}(x)\right) \mathrm{d} x \mid \\
& \leq\left(\left\|a\left(\cdot, \nabla \varphi_{J}\right)\right\|_{L^{p^{\prime}}(\Omega)^{d}}+\|f\|_{L^{p^{\prime}}(\Omega)}\right) \varepsilon \\
& \leq\left(\|b\|_{L^{p^{\prime}(\Omega)}}+\Lambda\left\|\nabla \varphi_{J}\right\|_{L^{p}(\Omega)^{d}}^{p-1}+\|f\|_{L^{p^{\prime}}(\Omega)}\right) \varepsilon \\
& \leq C_{18} \varepsilon
\end{aligned}
$$

where $C_{18}$ only depends on $p, b, \Lambda, f$ and $\bar{u}$ (we have used the bound $\left\|\nabla \varphi_{J}\right\|_{L^{p}(\Omega)^{d}} \leq 1+\|\nabla \bar{u}\|_{L^{p}(\Omega)^{d}}$ ). Hence, if $\operatorname{size}(\mathcal{D})$ is small enough, $\int_{\Omega}\left(a\left(x,\left(\nabla \varphi_{J}\right)_{\mathcal{D}}(x)\right)-a(x, \mathbf{v}(x))\right) \cdot\left(\left(\nabla \varphi_{J}\right)_{\mathcal{D}}(x)-\mathbf{v}(x)\right) \mathrm{d} x \leq C_{18} \varepsilon+\varepsilon$ and $(4.27)$ gives

$$
\int_{\Omega}(a(x, \nabla \bar{u}(x))-a(x, \mathbf{v}(x))) \cdot(\nabla \bar{u}(x)-\mathbf{v}(x)) \mathrm{d} x \leq\left(C_{18}+3\right) \varepsilon .
$$

Since the integrand is nonnegative (see $(1.4))$, this proves that $(a(\cdot, \nabla \bar{u})-a(\cdot, \mathbf{v})) \cdot(\nabla \bar{u}-\mathbf{v}) \rightarrow 0$ in $L^{1}(\Omega)$ as $\operatorname{size}(\mathcal{D}) \rightarrow 0$. Up to a subsequence, the convergence therefore also holds almost everywhere on $\Omega$. Let $x$ be such that, along this subsequence,

$$
(a(x, \nabla \bar{u}(x))-a(x, \mathbf{v}(x))) \cdot(\nabla \bar{u}(x)-\mathbf{v}(x)) \rightarrow 0 \quad \text { as } \operatorname{size}(\mathcal{D}) \rightarrow 0 .
$$

We have, by (1.3) and (1.5),

$$
\begin{aligned}
\alpha_{0}|\mathbf{v}(x)|^{p} \leq & a(x, \mathbf{v}(x)) \cdot \mathbf{v}(x) \\
= & (a(x, \nabla \bar{u}(x))-a(x, \mathbf{v}(x))) \cdot(\nabla \bar{u}(x)-\mathbf{v}(x))-a(x, \nabla \bar{u}(x)) \cdot(\nabla \bar{u}(x)-\mathbf{v}(x)) \\
& +a(x, \mathbf{v}(x)) \cdot \nabla \bar{u}(x) \\
\leq & (a(x, \nabla \bar{u}(x))-a(x, \mathbf{v}(x))) \cdot(\nabla \bar{u}(x)-\mathbf{v}(x))+C_{19}+C_{19}|\mathbf{v}(x)| \\
& +C_{19}|b(x)|+C_{19} \Lambda|\mathbf{v}(x)|^{p-1}
\end{aligned}
$$


where $C_{19}$ depends on $\bar{u}$ and $x$ but not on $\mathbf{v}$. The first term of this right-hand side being bounded (it tends to 0 ), this inequality shows that $\mathbf{v}(x)$ stays bounded as $\operatorname{size}(\mathcal{D}) \rightarrow 0$ (the power of $|\mathbf{v}(x)|$ in the left-hand side is greater than the powers in the right-hand side). Let $\xi$ be the limit of a subsequence of $\mathbf{v}(x)$; passing to the limit (along this new subsequence) in (4.28), thanks to $(1.2)$, we obtain $(a(x, \nabla \bar{u}(x))-a(x, \xi)) \cdot(\nabla \bar{u}(x)-\xi)=0$ and thus, by (1.4), $\xi=\nabla \bar{u}(x)$; since this is true for all converging subsequences of $\mathbf{v}(x)$, this shows that, along the subsequence for which (4.28) holds almost everywhere on $\Omega$, we have $\mathbf{v}(x) \rightarrow \nabla \bar{u}(x)$.

We can now conclude that $\mathbf{v} \rightarrow \nabla \bar{u}$ strongly in $L^{p}(\Omega)^{d}$. We first notice that

$$
\int_{\Omega} a(x, \mathbf{v}(x)) \cdot \mathbf{v}(x) \mathrm{d} x=\sum_{K \in \mathcal{M}} \mathrm{m}(K) a_{K}\left(\mathbf{v}_{K}\right) \cdot \mathbf{v}_{K}
$$

so that (4.25) implies, since $\bar{u}$ is a weak solution to (1.1),

$$
\limsup _{\operatorname{size}(\mathcal{D}) \rightarrow 0} \int_{\Omega} a(x, \mathbf{v}(x)) \cdot \mathbf{v}(x) \mathrm{d} x \leq \int_{\Omega} f(x) \bar{u}(x) \mathrm{d} x=\int_{\Omega} a(x, \nabla \bar{u}(x)) \cdot \nabla \bar{u}(x) \mathrm{d} x .
$$

Using (1.2) and the fact that $\mathbf{v} \rightarrow \nabla \bar{u}$ almost everywhere, we have $a(\cdot, \mathbf{v}) \cdot \mathbf{v} \rightarrow a(\cdot, \nabla \bar{u}) \cdot \nabla \bar{u}$ a.e. on $\Omega$ as $\operatorname{size}(\mathcal{D}) \rightarrow 0$; these functions being nonnegative (see (1.3)), Fatou's lemma gives

$$
\int_{\Omega} a(x, \nabla \bar{u}(x)) \cdot \nabla \bar{u}(x) \mathrm{d} x \leq \liminf _{\operatorname{size}(\mathcal{D}) \rightarrow 0} \int_{\Omega} a(x, \mathbf{v}(x)) \cdot \mathbf{v}(x) \mathrm{d} x .
$$

Gathering (4.29) and (4.30), we deduce

$$
\int_{\Omega} a(x, \mathbf{v}(x)) \cdot \mathbf{v}(x) \mathrm{d} x \rightarrow \int_{\Omega} a(x, \nabla \bar{u}(x)) \cdot \nabla \bar{u}(x) \mathrm{d} x \quad \text { as } \operatorname{size}(\mathcal{D}) \rightarrow 0 .
$$

Since the integrand $a(\cdot, \mathbf{v}) \cdot \mathbf{v}$ is nonnegative and converges a.e. to $a(\cdot, \nabla \bar{u}) \cdot \nabla \bar{u} \in L^{1}(\Omega)$, we see by Lemma 8.4 that $a(\cdot, \mathbf{v}) \cdot \mathbf{v}$ converges in $L^{1}(\Omega)$ as $\operatorname{size}(\mathcal{D}) \rightarrow 0$; in particular, $a(\cdot, \mathbf{v}) \cdot \mathbf{v}$ is equi-integrable and (1.3) thus shows that $|\mathbf{v}|^{p}$ is also equi-integrable. Vitali theorem then concludes the proof.

Remark 4.1. If the sequence of discretizations satisfies: there exists $C>0$ such that for all $n \geq 1$ and all $(K, L) \in \mathcal{M}_{n}$ neighboring control volumes we have $\frac{\operatorname{diam}(K)}{\operatorname{diam}(L)} \leq C$, then we can prove the strong convergence in $L^{p}(\Omega)$ of $u_{n}$ to $\bar{u}$ (by strengthening Lem. 3.1, see [13]). However, such an hypothesis restricts the sequences of discretizations we can choose (for example, if we want to refine the grids, it must be done by layers).

\section{More SCHEMES}

\subsection{Non-monotone operators}

We can apply, with minor modifications, the scheme of Section 2 to non-monotone equations of the kind

$$
\begin{cases}-\operatorname{div}(a(x, \bar{u}, \nabla \bar{u}))=f & \text { in } \Omega \\ \bar{u}=0 & \text { on } \partial \Omega\end{cases}
$$

where $f$ satisfies (1.6) and

$$
a: \Omega \times \mathbb{R} \times \mathbb{R}^{d} \rightarrow \mathbb{R}^{d} \text { is measurable w.r.t. its first variable }
$$
and continuous w.r.t. its last two variables,

$$
\exists \alpha_{0}>0 \text { such that } a(x, s, \xi) \cdot \xi \geq \alpha_{0}|\xi|^{p} \text { for a.e. } x \in \Omega \text {, all } s \in \mathbb{R} \text { and all } \xi \in \mathbb{R}^{d}
$$


1086

$$
\begin{gathered}
(a(x, s, \xi)-a(x, s, \eta)) \cdot(\xi-\eta)>0 \text { for a.e. } x \in \Omega \text {, all } s \in \mathbb{R} \text { and all } \xi \neq \eta, \\
\exists b \in L^{p^{\prime}}(\Omega), \exists r \in\left[1, p\left[, \exists \Lambda>0 \text { such that }|a(x, s, \xi)| \leq b(x)+\Lambda|s|^{r-1}+\Lambda|\xi|^{p-1},\right.\right. \\
\text { for a.e. } x \in \Omega \text {, all } s \in \mathbb{R} \text { and all } \xi \in \mathbb{R}^{d} .
\end{gathered}
$$

Under these assumptions, there exists at least one solution to (5.1). Except in some special cases (see e.g. [6]), uniqueness is lost.

The scheme we consider is (2.2), (2.3), (2.5) and the following natural replacement of (2.4):

$$
\mathrm{m}(K) a_{K}\left(u_{K}, \mathbf{v}_{K}\right)=\sum_{\sigma \in \mathcal{E}_{K}} F_{K, \sigma}\left(\mathbf{x}_{\sigma}-\mathbf{x}_{K}\right), \quad \forall K \in \mathcal{M},
$$

where $a_{K}(s, \xi)=\frac{1}{\mathrm{~m}(K)} \int_{K} a(x, s, \xi) \mathrm{d} x$.

The space $L_{p, \nu}(\mathcal{D})$ does not change (it was solely based on (2.2)) and we can therefore use Lemmas $3.1,3.2$ and 3.3. Existence of a solution to $((2.2),(2.3),(2.5),(5.6))$ is obtained exactly as in the proof of Theorem 2.1; however, in general, uniqueness of the solution fails (as for (5.1)).

The mixed finite volume scheme for (5.1) then converges according to the following result. Notice that, since there is not uniqueness of the solution to the limit problem, we can only prove the convergence of a subsequence of approximations.

Theorem 5.1. Assume Hypotheses (5.2)-(5.5) and (1.6). Let $\left(\mathcal{D}_{n}\right)_{n \geq 1}$ be a sequence of admissible discretizations of $\Omega$ such that $\operatorname{size}\left(\mathcal{D}_{n}\right) \rightarrow 0$ and $\left(\operatorname{regul}\left(\mathcal{D}_{n}\right)\right)_{n \geq 1}$ is bounded. Let $\nu_{0}>0$ and $\left.\beta \in\right]-p^{\prime}(d-1),-p^{\prime}(d-2)[$. Let $\left(u_{n}, \mathbf{v}_{n}, F_{n}\right)$ be a solution to $((2.2),(2.3),(2.5),(5.6))$ for $\mathcal{D}=\mathcal{D}_{n}$ and $\nu_{K}=\nu_{0} \operatorname{diam}(K)^{\beta}$ for all $K \in \mathcal{M}_{n}$.

Then there exists a weak solution $\bar{u} \in W_{0}^{1, p}(\Omega)$ to (5.1) such that, up to a subsequence, $u_{n} \rightarrow \bar{u}$ weakly in $L^{p}(\Omega)$ and strongly in $L^{q}(\Omega)$ for all $q<p$, and $\mathbf{v}_{n} \rightarrow \nabla \bar{u}$ strongly in $L^{p}(\Omega)^{d}$.

Proof of Theorem 5.1. We drop the indices $n$. Under the assumptions on $\mathcal{D}$ and $\nu$, we obtain the same estimate on the solutions to $((2.2),(2.3),(2.5),(5.6))$ as in Lemma 4.1 (using (5.3) instead of (1.3)). Thus, as in Step 1 of the proof of Theorem 2.2, we have a subsequence of $(u, \mathbf{v})$ which converges, as $\operatorname{size}(\mathcal{D}) \rightarrow 0$ and $\operatorname{regul}(\mathcal{D}) \operatorname{stays}$ bounded, to some $(\bar{u}, \nabla \bar{u})$ weakly in $L^{p}(\Omega)^{d+1}$, with $\bar{u} \in W_{0}^{1, p}(\Omega)$. We also have $u \rightarrow \bar{u}$ strongly in $L^{q}(\Omega)$ for all $q<p$ and therefore, up to another subsequence, a.e. on $\Omega$.

To prove that $\bar{u}$ is a solution to (5.1), we replace (4.19) by

$$
0 \leq \sum_{K \in \mathcal{M}} \mathrm{m}(K)\left(a_{K}\left(u_{K},[\nabla \varphi]_{K}\right)-a_{K}\left(u_{K}, \mathbf{v}_{K}\right)\right) \cdot\left([\nabla \varphi]_{K}-\mathbf{v}_{K}\right)
$$

(see (5.4)) and the only term whose study of convergence differs from what is done in the proof of Theorem 2.2 is

$$
\sum_{K \in \mathcal{M}} \mathrm{m}(K) a_{K}\left(u_{K},[\nabla \varphi]_{K}\right) \cdot\left([\nabla \varphi]_{K}-\mathbf{v}_{K}\right)=\int_{\Omega} a_{\mathcal{D}}\left(x, u(x),(\nabla \varphi)_{\mathcal{D}}(x)\right) \cdot\left((\nabla \varphi)_{\mathcal{D}}(x)-\mathbf{v}(x)\right) \mathrm{d} x
$$

(where $a_{\mathcal{D}}(x, s, \xi)=a_{K}(s, \xi)$ if $\left.x \in K\right)$. To prove the convergence of this term to $\int_{\Omega} a(x, \bar{u}(x), \nabla \varphi(x)) \cdot(\nabla \varphi(x)-$ $\nabla \bar{u}(x)) \mathrm{d} x$, we only need to prove that $a_{\mathcal{D}}\left(\cdot, u,(\nabla \varphi)_{\mathcal{D}}\right) \rightarrow a(\cdot, \bar{u}, \nabla \varphi)$ strongly in $L^{p^{\prime}}(\Omega)^{d}$ as $\operatorname{size}(\mathcal{D}) \rightarrow 0($ since $(\nabla \varphi)_{\mathcal{D}} \rightarrow \nabla \varphi$ and $\mathbf{v} \rightarrow \nabla \bar{u}$ weakly in $\left.L^{p}(\Omega)^{d}\right)$. This is done using the same techniques as in the proof of Lemma 8.3: define $(a(\cdot, \bar{u}, \nabla \varphi))_{\mathcal{D}}$ as the piecewise constant function whose value on a control volume $K$ is the mean value of $a(\cdot, \bar{u}, \nabla \varphi)$ on $K$; we have, the same way we prove (8.1),

$$
\int_{\Omega}\left|a_{\mathcal{D}}\left(x, u(x),(\nabla \varphi)_{\mathcal{D}}(x)\right)-(a(\cdot, \bar{u}, \nabla \varphi))_{\mathcal{D}}(x)\right|^{p^{\prime}} \mathrm{d} x \leq \int_{\Omega}\left|a\left(y, u(y),(\nabla \varphi)_{\mathcal{D}}(y)\right)-a(y, \bar{u}(y), \nabla \varphi(y))\right|^{p^{\prime}} \mathrm{d} y
$$

For a.e. $y \in \Omega$ and as $\operatorname{size}(\mathcal{D}) \rightarrow 0$, we have $(\nabla \varphi)_{\mathcal{D}}(y) \rightarrow \nabla \varphi(y)$ and $u(y) \rightarrow \bar{u}(y)$ so that, by (5.2), $\left|a\left(y, u(y),(\nabla \varphi)_{\mathcal{D}}(y)\right)-a(y, \bar{u}(y), \nabla \varphi(y))\right|^{p^{\prime}} \rightarrow 0$. By (5.5), we can write

$$
\left|a\left(y, u(y),(\nabla \varphi)_{\mathcal{D}}(y)\right)-a(y, \bar{u}(y), \nabla \varphi(y))\right|^{p^{\prime}} \leq C_{20} b(y)^{p^{\prime}}+C_{20}\|\nabla \varphi\|_{\infty}^{p}+C_{20}|u(y)|^{p^{\prime}(r-1)}+C_{20}|\bar{u}(y)|^{p^{\prime}(r-1)}
$$


where $C_{20}$ only depends on $p$ and $\Lambda$. But $0 \leq p^{\prime}(r-1)<p^{\prime}(p-1)=p$ and $u$ is bounded in $L^{p}(\Omega)$; hence, $|u|^{p^{\prime}(r-1)}$ is equi-integrable and Vitali theorem shows that the right-hand side of $(5.8)$ tends to 0 as $\operatorname{size}(\mathcal{D}) \rightarrow 0$. As $a(\cdot, \bar{u}, \nabla \varphi) \in L^{p^{\prime}}(\Omega)^{d}$, we have $(a(\cdot, \bar{u}, \nabla \varphi))_{\mathcal{D}} \rightarrow a(\cdot, \bar{u}, \nabla \varphi)$ in $L^{p^{\prime}}(\Omega)^{d}$ as size $(\mathcal{D}) \rightarrow 0$, which concludes the proof of the convergence of $a_{\mathcal{D}}\left(\cdot, u,(\nabla \varphi)_{\mathcal{D}}\right)$ to $a(\cdot, \bar{u}, \nabla \varphi)$ in $L^{p^{\prime}}(\Omega)^{d}$.

Passing to the limsup in (5.7), we arrive at the equivalent of $(4.26)$ :

$$
0 \leq \int_{\Omega} a(x, \bar{u}(x), \nabla \varphi(x)) \cdot(\nabla \varphi(x)-\nabla \bar{u}(x)) \mathrm{d} x-\int_{\Omega} f(x)(\varphi(x)-\bar{u}(x)) \mathrm{d} x
$$

and the conclusion that $\bar{u}$ is a weak solution to (5.1) follows as in the proof of Theorem 2.2.

To obtain the strong convergence of $\mathbf{v}$ to $\nabla \bar{u}$, we reason as in Step 3 of the proof of Theorem 2.2 with $a(\cdot, u, \cdot)$ instead of $a(\cdot, \cdot)$ : taking $\left(\varphi_{j}\right)_{j \geq 1} \in C_{c}^{\infty}(\Omega)$ which converges to $\bar{u}$ in $W_{0}^{1, p}(\Omega)$, we write

$$
\begin{aligned}
\int_{\Omega}(a(x, u(x), \nabla \bar{u}(x))- & a(x, u(x), \mathbf{v}(x))) \cdot(\nabla \bar{u}(x)-\mathbf{v}(x)) \mathrm{d} x \\
\leq & \left\|a(\cdot, u, \nabla \bar{u})-a\left(\cdot, u, \nabla \varphi_{j}\right)\right\|_{L^{p^{\prime}}(\Omega)^{d}}\left(\|\nabla \bar{u}\|_{L^{p}(\Omega)^{d}}+\|\mathbf{v}\|_{L^{p}(\Omega)^{d}}\right) \\
& \quad+\int_{\Omega}\left(a\left(x, u(x), \nabla \varphi_{j}(x)\right)-a(x, u(x), \mathbf{v}(x))\right) \cdot(\nabla \bar{u}(x)-\mathbf{v}(x)) \mathrm{d} x \\
\leq & \left\|a(\cdot, u, \nabla \bar{u})-a\left(\cdot, u, \nabla \varphi_{j}\right)\right\|_{L^{p^{\prime}}(\Omega)^{d}}\left(\|\nabla \bar{u}\|_{L^{p}(\Omega)^{d}}+\|\mathbf{v}\|_{L^{p}(\Omega)^{d}}\right) \\
& +\left(\left\|a\left(\cdot, u, \nabla \varphi_{j}\right)\right\|_{L^{p^{\prime}}(\Omega)^{d}}+\|a(\cdot, u, \mathbf{v})\|_{L^{p^{\prime}}(\Omega)^{d}}\right)\left\|\nabla \bar{u}-\nabla \varphi_{j}\right\|_{L^{p}(\Omega)^{d}} \\
& +\int_{\Omega}\left(a\left(x, u(x), \nabla \varphi_{j}(x)\right)-a(x, u(x), \mathbf{v}(x))\right) \cdot\left(\nabla \varphi_{j}(x)-\mathbf{v}(x)\right) \mathrm{d} x
\end{aligned}
$$

Since $u \rightarrow \bar{u}$ a.e. on $\Omega$ and $|u|^{p^{\prime}(r-1)}$ is equi-integrable as size $(\mathcal{D}) \rightarrow 0$, and since $\nabla \varphi_{j} \rightarrow \nabla \bar{u}$ in $L^{p}(\Omega)^{d}$ as $j \rightarrow \infty$, it is possible to prove (for example by way of contradiction), using (5.2) and (5.5), that $a\left(\cdot, u, \nabla \varphi_{j}\right) \rightarrow a(\cdot, u, \nabla \bar{u})$ in $L^{p^{\prime}}(\Omega)^{d}$ as $j \rightarrow \infty$, uniformly with respect to $u$ and thus $\mathcal{D}$ (recall that, though we do not indicate it, $u$ and $\mathcal{D}$ are considered along a sequence such that $\operatorname{size}(\mathcal{D}) \rightarrow 0$ ). Therefore, we can take $j=J$ not depending on $\mathcal{D}$ such that $\left\|\varphi_{J}-\bar{u}\right\|_{W_{0}^{1, p}(\Omega)} \leq \varepsilon$ and

$$
\begin{aligned}
\int_{\Omega}(a(x, u(x), \nabla \bar{u}(x))-a(x, u(x), \mathbf{v}(x))) \cdot( & \nabla \bar{u}(x)-\mathbf{v}(x)) \mathrm{d} x \\
& \leq \varepsilon+\int_{\Omega}\left(a\left(x, u(x), \nabla \varphi_{J}(x)\right)-a(x, u(x), \mathbf{v}(x))\right) \cdot\left(\nabla \varphi_{J}(x)-\mathbf{v}(x)\right) \mathrm{d} x
\end{aligned}
$$

Still using the fact that $u$ converges a.e. and $|u|^{p^{\prime}(r-1)}$ is equi-integrable as size $(\mathcal{D}) \rightarrow 0$, we see that $a\left(\cdot, u,\left(\nabla \varphi_{J}\right)_{\mathcal{D}}\right)-a\left(\cdot, u, \nabla \varphi_{J}\right) \rightarrow 0$ in $L^{p^{\prime}}(\Omega)^{d}$ as $\operatorname{size}(\mathcal{D}) \rightarrow 0$ and we arrive at the equivalent of (4.27): for $\operatorname{size}(\mathcal{D})$ small enough,

$$
\begin{aligned}
& \int_{\Omega}(a(x, u(x), \nabla \bar{u}(x))-a(x, u(x),\mathbf{v}(x))) \cdot(\nabla \bar{u}(x)-\mathbf{v}(x)) \mathrm{d} x \\
& \leq 2 \varepsilon+\int_{\Omega}\left(a\left(x, u(x),\left(\nabla \varphi_{J}\right)_{\mathcal{D}}\right)(x)-a(x, u(x), \mathbf{v}(x))\right) \cdot\left(\left(\nabla \varphi_{J}\right)_{\mathcal{D}}(x)-\mathbf{v}(x)\right) \mathrm{d} x
\end{aligned}
$$

This last term is the right-hand side of (5.7) with $\varphi=\varphi_{J}$, and the reasoning at the beginning of the proof shows that it is bounded from above by a quantity which tends to $\int_{\Omega} a\left(x, \bar{u}(x), \nabla \varphi_{J}(x)\right) \cdot\left(\nabla \varphi_{J}(x)-\nabla \bar{u}(x)\right) \mathrm{d} x-$ $\int_{\Omega} f(x)\left(\varphi_{J}(x)-\bar{u}(x)\right) \mathrm{d} x$ as $\operatorname{size}(\mathcal{D}) \rightarrow 0$. We deduce, as in the proof of Theorem 2.2 , that $(a(\cdot, u, \nabla \bar{u})-a(\cdot, u, \mathbf{v}))$. 
$(\nabla \bar{u}-\mathbf{v}) \rightarrow 0$ in $L^{1}(\Omega)$ as $\operatorname{size}(\mathcal{D}) \rightarrow 0$, which gives, up to a subsequence, the convergence a.e. of $\mathbf{v}$ to $\nabla \bar{u}$. We have

$$
\int_{\Omega} a(x, u(x), \mathbf{v}(x)) \cdot \mathbf{v}(x) \mathrm{d} x=\sum_{K \in \mathcal{M}} \mathrm{m}(K) a_{K}\left(u_{K}, \mathbf{v}_{K}\right) \cdot \mathbf{v}_{K} \leq \int_{\Omega} f(x) u(x) \mathrm{d} x
$$

(this inequality, obtained as the one in (4.25), was already necessary to take the limsup of (5.7)). Hence,

$$
\limsup _{\operatorname{size}(\mathcal{D}) \rightarrow 0} \int_{\Omega} a(x, u(x), \mathbf{v}(x)) \cdot \mathbf{v}(x) \mathrm{d} x \leq \int_{\Omega} f(x) \bar{u}(x) \mathrm{d} x=\int_{\Omega} a(x, \bar{u}(x), \nabla \bar{u}(x)) \cdot \nabla \bar{u}(x) \mathrm{d} x
$$

and, using the a.e. convergences of $u$ and v, Fatou's lemma implies

$$
\int_{\Omega} a(x, \bar{u}(x), \nabla \bar{u}(x)) \cdot \nabla \bar{u}(x) \mathrm{d} x \leq \liminf _{\operatorname{size}(\mathcal{D}) \rightarrow 0} \int_{\Omega} a(x, u(x), \mathbf{v}(x)) \cdot \mathbf{v}(x) \mathrm{d} x .
$$

As in the proof of Theorem 2.2, we then obtain the convergence of $a(\cdot, u, \mathbf{v}) \cdot \mathbf{v}$ in $L^{1}(\Omega)$, and thus its equiintegrability as $\operatorname{size}(\mathcal{D}) \rightarrow 0$. (5.3) therefore gives the equi-integrability of $|\mathbf{v}|^{p}$ and concludes the proof.

\subsection{Non-penalized scheme}

We can also study a non-penalized version of the scheme of Section 2, that is to say the scheme for (1.1) defined by (2.3), (2.4), (2.5) and

$$
\begin{gathered}
\mathbf{v}_{K} \cdot\left(\mathbf{x}_{\sigma}-\mathbf{x}_{K}\right)+\mathbf{v}_{L} \cdot\left(\mathbf{x}_{L}-\mathbf{x}_{\sigma}\right)=u_{L}-u_{K}, \quad \forall \sigma=K \mid L \in \mathcal{E}_{\text {int }} \\
\mathbf{v}_{K} \cdot\left(\mathbf{x}_{\sigma}-\mathbf{x}_{K}\right)=-u_{K}, \quad \forall K \in \mathcal{M}, \forall \sigma \in \mathcal{E}_{K} \cap \mathcal{E}_{\text {ext }}
\end{gathered}
$$

(i.e. (2.2) with $\nu_{K}=0$ ). For general discretizations, it is not possible to prove a priori estimates on the solutions to $((2.3)-(2.5),(5.9))^{1}$. However, restricting ourselves to "simplicial discretizations" as defined below, we can prove the existence and uniqueness of an approximate solution, and its convergence to the weak solution of (1.1).

Definition 5.1. We say that an admissible discretization $\mathcal{D}$ of $\Omega$ is simplicial if, for all $K \in \mathcal{M}, K$ is the interior of the convex hull of $d+1$ points of $\mathbb{R}^{d}$ in general position (not contained in an affine hyperplane) and $\operatorname{Card}\left(\mathcal{E}_{K}\right)=d+1$ (the smallest possible number of edges for a control volume).

Remark 5.1. In dimension $d=2$, simplicial discretizations are the ones made of triangles.

Theorem 5.2. Assume Hypotheses (1.2)-(1.6). Let $\left(\mathcal{D}_{n}\right)_{n \geq 1}$ be a sequence of admissible discretizations of $\Omega$ such that $\operatorname{size}\left(\mathcal{D}_{n}\right) \rightarrow 0$ and $\left(\operatorname{regul}\left(\mathcal{D}_{n}\right)\right)_{n \geq 1}$ is bounded. We also assume that each discretization $\mathcal{D}_{n}$ is simplicial.

Then, for all $n \geq 1$, there exists a unique solution $\left(u_{n}, \mathbf{v}_{n}, F_{n}\right)$ to $((2.3)-(2.5),(5.9))$ for $\mathcal{D}=\mathcal{D}_{n}$. Moreover, if $\bar{u} \in W_{0}^{1, p}(\Omega)$ is the weak solution to $(1.1)$ then, as $n \rightarrow \infty, u_{n} \rightarrow \bar{u}$ weakly in $L^{p}(\Omega)$ and strongly in $L^{q}(\Omega)$ for all $q<p$, and $\mathbf{v}_{n} \rightarrow \nabla \bar{u}$ strongly in $L^{p}(\Omega)^{d}$.

Remark 5.2. We can also, in the case of simplicial discretizations, prove the convergence of a non-penalized version of the scheme presented in Subsection 5.1 for non-monotone operators.

Remark 5.3. In fact, sequences of simplicial discretizations $\left(\mathcal{D}_{n}\right)_{n \geq 1}$ such that $\left(\operatorname{regul}\left(\mathcal{D}_{n}\right)\right)_{n \geq 1}$ is bounded satisfy the assumption in Remark 4.1. In Theorem 5.2, the convergence of $\left(u_{n}\right)_{n \geq 1}$ to $\bar{u}$ is therefore strong in $L^{p}(\Omega)$.

\footnotetext{
${ }^{1}$ Consider the linear case on cartesian meshes; take one control volume $K$ and put $F_{K, \sigma}=+1$ on two parallel sides and -1 on the other two sides; extend then these fluxes by conservativity: this gives an element in the kernel of the system. Hence, the fluxes cannot be estimated; however, one can see that estimates on $u$ and $\mathbf{v}$ still hold, but the lack of estimates on the fluxes prevents to pass to the limit.
} 
Proof of Theorem 5.2. Notice first that, though the space $L_{p}(\mathcal{D})$, defined as the set of functions $(u, \mathbf{v}) \in H_{\mathcal{D}} \times H_{\mathcal{D}}^{d}$ which satisfy $(5.9)$, is different from $L_{p, \nu}(\mathcal{D})$, one can verify that all the results of Section 3 are still true for $L_{p}(\mathcal{D})$ (even for discretizations not made of simplicial meshes), provided that we replace all the $\nu_{K}$ by 0 (in which case the terms $M_{1}$ and $M_{p}$ vanish and there is no mention of $F$ ).

As usual, we drop the indices $n$. If $F_{K}=\left(F_{K, \sigma}\right)_{\sigma \in \mathcal{E}_{K}}$ and $A_{K}$ is the $(d+1) \times(d+1)$ matrix whose columns are the vectors $\left(1, \mathbf{x}_{\sigma}-\mathbf{x}_{K}\right)^{T}\left(\sigma \in \mathcal{E}_{K}\right)$, then Equations $(2.4)-(2.5)$ can be written

$$
A_{K} F_{K}=\left(\begin{array}{c}
-\int_{K} f(x) \mathrm{d} x \\
\operatorname{m}(K) a_{K}\left(\mathbf{v}_{K}\right)
\end{array}\right)
$$

It is shown in [12], proof of Lemma 6.4 , that $A_{K}$ is invertible and that

$$
\left\|A_{K}^{-1}\right\| \leq C_{21} \sup \left(1, \operatorname{diam}(K)^{-1}\right)
$$

with $C_{21}$ only depending on $d$ and a bound on $\operatorname{regul}(\mathcal{D})$ (recall that there are exactly $d+1$ edges $\sigma \in \mathcal{E}_{K}$, so the norms we choose on the spaces $\mathbb{R}^{\operatorname{Card}\left(\mathcal{E}_{K}\right)}=\mathbb{R}^{d+1}$ involved do not matter; in the following, we take the norms $\left\|F_{K}\right\|=\left(\sum_{\sigma \in \mathcal{E}_{K}}\left|F_{K, \sigma}\right|^{p^{\prime}}\right)^{1 / p^{\prime}}$ and $\left.\left\|\left(-\int_{K} f(x) \mathrm{d} x, \mathrm{~m}(K) a_{K}\left(\mathbf{v}_{K}\right)\right)^{T}\right\|=\left|\int_{K} f(x) \mathrm{d} x\right|+\left|\mathrm{m}(K) a_{K}\left(\mathbf{v}_{K}\right)\right|\right)$.

Step 1. Existence and uniqueness of an approximate solution.

Thanks to (5.10), we can eliminate the fluxes in the non-linear system $((2.3)-(2.5),(5.9))$, which can therefore be considered as a system on $(u, \mathbf{v})$. Existence of a solution is obtained, as in the proof of Theorem 2.1, through a priori estimates on this system where $a_{K}\left(\mathbf{v}_{K}\right)$ has been replaced by $t a_{K}\left(\mathbf{v}_{K}\right)+(1-t) \mathbf{v}_{K}$. Reasoning as in Step 1 of the proof of Theorem 2.1, we arrive at (4.12) which reads, since $\nu_{K}=0$,

$$
\lambda_{\mathcal{D}}\left(t \alpha_{0}\left\|\left(\mathbf{v}_{K}\right)\right\|_{l^{1}}^{p}+(1-t)\left\|\left(\mathbf{v}_{K}\right)\right\|_{l^{1}}^{2}\right) \leq C_{7}\left\|\left(u_{K}\right)\right\|_{l^{1}}
$$

In our framework, Lemma 3.1 gives $\|u\|_{L^{p}(\Omega)} \leq C_{1}\|\mathbf{v}\|_{L^{p}(\Omega)^{d}}$, and we thus deduce an estimate (independent on $t$ ) on $\mathbf{v}$ which gives, in turn, an estimate on $u$. Existence of a solution to $((2.3)-(2.5),(5.9))$ follows from these estimates and the topological degree.

Uniqueness is done exactly as in Step 2 of the proof of Theorem 2.1: even with $\nu_{K}=0$, the same inequalities implies $\mathbf{v}=\mathbf{v}^{\prime}$ which gives, by (5.9), $u=u^{\prime}$ and, using (5.10), $F=F^{\prime}$.

Step 2. Convergence of the approximate solution.

The estimate of Lemma 4.1 is still true for $\nu_{K}=0$ (recall that, in this case, Lem. 3.1 bounds $\|u\|_{L^{p}(\Omega)}$ solely in terms of $\left.\|\mathbf{v}\|_{L^{p}(\Omega)^{d}}\right)$. This shows that $\mathbf{v}$ is bounded in $L^{p}(\Omega)^{d}$ and, applying Lemma 3.3 with $\nu_{K}=0$, we find $\bar{u} \in W_{0}^{1, p}(\Omega)$ such that, up to a subsequence and as $\operatorname{size}(\mathcal{D}) \rightarrow 0, u \rightarrow \bar{u}$ weakly in $L^{p}(\Omega)$ and strongly in $L^{q}(\Omega)$ for all $q<p$, and $\mathbf{v} \rightarrow \nabla \bar{u}$ weakly in $L^{p}(\Omega)^{d}$.

To prove that $\bar{u}$ is a weak solution to (1.1), we use the same argument as in the proof of Theorem 2.2 . Starting from (4.19), the only term whose convergence needs a special work is $\sum_{K \in \mathcal{M}} \mathrm{m}(K) a_{K}\left(\mathbf{v}_{K}\right) \cdot[\nabla \varphi]_{K}$,

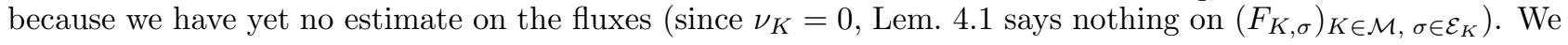
still write

$$
\sum_{K \in \mathcal{M}} \mathrm{m}(K) a_{K}\left(\mathbf{v}_{K}\right) \cdot[\nabla \varphi]_{K}=\sum_{K \in \mathcal{M}} \varphi\left(\mathbf{x}_{K}\right) \int_{K} f(x) \mathrm{d} x+T_{7}
$$


with $\left|T_{7}\right| \leq C_{17} \sum_{K \in \mathcal{M}} \sum_{\sigma \in \mathcal{E}_{K}} \operatorname{diam}(K)^{2}\left|F_{K, \sigma}\right|$. By $(2.1)$,

$$
\begin{aligned}
\left|T_{7}\right| & \leq C_{17} \sum_{K \in \mathcal{M}} \sum_{\sigma \in \mathcal{E}_{K}} \operatorname{diam}(K)^{2} \mathrm{~m}(K)^{-\frac{1}{p}}\left|F_{K, \sigma}\right| \mathrm{m}(K)^{\frac{1}{p}} \\
& \leq C_{17}\left(\sum_{K \in \mathcal{M}} \sum_{\sigma \in \mathcal{E}_{K}} \operatorname{diam}(K)^{2 p^{\prime}} \mathrm{m}(K)^{-\frac{p^{\prime}}{p}}\left|F_{K, \sigma}\right|^{p^{\prime}}\right)^{\frac{1}{p}}\left(\sum_{K \in \mathcal{M}} \sum_{\sigma \in \mathcal{E}_{K}} \mathrm{~m}(K)\right)^{\frac{1}{p}} \\
& \leq C_{17} \operatorname{regul}(\mathcal{D})^{\frac{1}{p}} \mathrm{~m}(\Omega)^{\frac{1}{p}}\left(\frac{\operatorname{regul}(\mathcal{D})^{\frac{p^{\prime}}{p}}}{\omega_{d}^{\frac{p^{\prime}}{p}}} \sum_{K \in \mathcal{M}} \sum_{\sigma \in \mathcal{E}_{K}} \operatorname{diam}(K)^{2 p^{\prime}-d \frac{p^{\prime}}{p}}\left|F_{K, \sigma}\right|^{p^{\prime}}\right)^{\frac{1}{p^{\prime}}} .
\end{aligned}
$$

By (5.10) and (5.11) we have, taking size $(\mathcal{D}) \leq 1$ and using (1.5),

$$
\begin{aligned}
\left(\sum_{\sigma \in \mathcal{E}_{K}}\left|F_{K, \sigma}\right|^{p^{\prime}}\right)^{\frac{1}{p^{\prime}}}=\left\|F_{K}\right\| & \leq C_{21} \operatorname{diam}(K)^{-1}\left(\int_{K}|f(x)| \mathrm{d} x+\mathrm{m}(K)\left|a_{K}\left(\mathbf{v}_{K}\right)\right|\right) \\
& \leq C_{21} \operatorname{diam}(K)^{-1}\left(\int_{K}|f(x)| \mathrm{d} x+\int_{K} b(x) \mathrm{d} x+\mathrm{m}(K) \Lambda\left|\mathbf{v}_{K}\right|^{p-1}\right) .
\end{aligned}
$$

Hence,

$$
\begin{aligned}
\sum_{\sigma \in \mathcal{E}_{K}}\left|F_{K, \sigma}\right|^{p^{\prime}} & \leq C_{22} \operatorname{diam}(K)^{-p^{\prime}}\left(\int_{K}\left(|f(x)|+b(x)+|\mathbf{v}(x)|^{p-1}\right) \mathrm{d} x\right)^{p^{\prime}} \\
& \leq C_{22} \operatorname{diam}(K)^{-p^{\prime}} \mathrm{m}(K)^{p^{\prime}-1} \int_{K}\left(|f(x)|+b(x)+|\mathbf{v}(x)|^{p-1}\right)^{p^{\prime}} \mathrm{d} x \\
& \leq C_{22} \omega_{d}^{p^{\prime}-1} \operatorname{diam}(K)^{d\left(p^{\prime}-1\right)-p^{\prime}} \int_{K}\left(|f(x)|+b(x)+|\mathbf{v}(x)|^{p-1}\right)^{p^{\prime}} \mathrm{d} x
\end{aligned}
$$

where $C_{22}$ only depends on $d, p, \Lambda$ and a bound on $\operatorname{regul}(\mathcal{D})$. (5.12) then implies

$$
\left|T_{7}\right| \leq C_{23}\left(\sum_{K \in \mathcal{M}} \operatorname{diam}(K)^{2 p^{\prime}-d \frac{p^{\prime}}{p}+d\left(p^{\prime}-1\right)-p^{\prime}} \int_{K}\left(|f(x)|+b(x)+|\mathbf{v}(x)|^{p-1}\right)^{p^{\prime}} \mathrm{d} x\right)^{\frac{1}{p^{\prime}}}
$$

where $C_{23}$ only depends on $\varphi, d, p, \Omega, \Lambda$ and a bound on $\operatorname{regul}(\mathcal{D})$. But $2 p^{\prime}-d \frac{p^{\prime}}{p}+d\left(p^{\prime}-1\right)-p^{\prime}=$ $p^{\prime}+d p^{\prime}\left(1-\frac{1}{p}\right)-d=p^{\prime}$, so that

$$
\begin{aligned}
\left|T_{7}\right| & \leq C_{23} \operatorname{size}(\mathcal{D})\left(\sum_{K \in \mathcal{M}} \int_{K}\left(|f(x)|+b(x)+|\mathbf{v}(x)|^{p-1}\right)^{p^{\prime}} \mathrm{d} x\right)^{\frac{1}{p^{\prime}}} \\
& =C_{23} \operatorname{size}(\mathcal{D})\left(\int_{\Omega}\left(|f(x)|+b(x)+|\mathbf{v}(x)|^{p-1}\right)^{p^{\prime}} \mathrm{d} x\right)^{\frac{1}{p^{\prime}}} .
\end{aligned}
$$

Since $\mathbf{v}$ is bounded in $L^{p}(\Omega)^{d},|\mathbf{v}|^{p-1}$ is bounded in $L^{p^{\prime}}(\Omega)$ and this inequality proves that $T_{7} \rightarrow 0$ as size $(\mathcal{D}) \rightarrow 0$. We then conclude as in the proof of Theorem 2.2. 


\subsection{Right-hand sides in $W^{-1, p^{\prime}}(\Omega)$}

The natural space for the right-hand side of $(1.1)$ is in fact $W^{-1, p^{\prime}}(\Omega)$ (see [22]); besides, such terms can naturally appear when obtaining conservative equations as (1.1) (see [19]). Following the ideas of [14], we can adapt our scheme to this setting.

If $f \in W^{-1, p^{\prime}}(\Omega)$, it is known that $f=\operatorname{div}(\mathbf{G})$ for some $\mathbf{G} \in L^{p^{\prime}}(\Omega)^{d}$. Let $\mathcal{D}$ be an admissible discretization of $\Omega$. For all $K \in \mathcal{M}$, we can formally write $\int_{K} f(x) \mathrm{d} x=\sum_{\sigma \in \mathcal{E}_{K}} \int_{\sigma} \mathbf{G} \cdot \mathbf{n}_{K, \sigma} \mathrm{d} \gamma$; defining

$$
\mathbf{G}_{\sigma}= \begin{cases}\frac{1}{\mathrm{~m}(K \cup L)} \int_{K \cup L} \mathbf{G}(x) \mathrm{d} x & \text { if } \sigma=K \mid L \in \mathcal{E}_{\mathrm{int}}, \\ \frac{1}{\mathrm{~m}(K)} \int_{K} \mathbf{G}(x) \mathrm{d} x & \text { if } \sigma \in \mathcal{E}_{\text {ext }} \cap \mathcal{E}_{K},\end{cases}
$$

the expression $\mathrm{m}(\sigma) \mathbf{G}_{\sigma} \cdot \mathbf{n}_{K, \sigma}$ can be seen as a replacement of $\int_{\sigma} \mathbf{G} \cdot \mathbf{n}_{K, \sigma} \mathrm{d} \gamma$ (which is not well defined) and the finite volume scheme for (1.1) with $f=\operatorname{div}(\mathbf{G})$ therefore reads: $(2.2)-(2.4)$ and

$$
-\sum_{\sigma \in \mathcal{E}_{K}} F_{K, \sigma}=\sum_{\sigma \in \mathcal{E}_{K}} \mathrm{~m}(\sigma) \mathbf{G}_{\sigma} \cdot \mathbf{n}_{K, \sigma}, \quad \forall K \in \mathcal{M}
$$

We then have the following result.

Theorem 5.3. Assume Hypotheses (1.2)-(1.5) and that $f=\operatorname{div}(\mathbf{G})$ with $\mathbf{G} \in L^{p^{\prime}}(\Omega)^{d}$. Let $\left(\mathcal{D}_{n}\right)_{n \geq 1}$ be a sequence of admissible discretizations of $\Omega$ such that $\operatorname{size}\left(\mathcal{D}_{n}\right) \rightarrow 0$ and $\left(\operatorname{regul}\left(\mathcal{D}_{n}\right)\right)_{n \geq 1}$ is bounded. Let $\nu_{0}>0$ and $\beta \in]-p^{\prime}(d-1),-p^{\prime}(d-2)[$.

Then, for all $n \geq 1$, there exists a unique solution $\left(u_{n}, \mathbf{v}_{n}, F_{n}\right)$ to $((2.2)-(2.4),(5.13))$ with $\mathcal{D}=\mathcal{D}_{n}$ and $\nu_{K}=\nu_{0} \operatorname{diam}(K)^{\beta}$ for all $K \in \mathcal{M}_{n}$.

Moreover, if $\bar{u} \in W_{0}^{1, p}(\Omega)$ is the weak solution to (1.1) then, as $n \rightarrow \infty, u_{n} \rightarrow \bar{u}$ weakly in $L^{p}(\Omega)$ and strongly in $L^{q}(\Omega)$ for all $q<p$, and $\mathbf{v}_{n} \rightarrow \nabla \bar{u}$ strongly in $L^{p}(\Omega)^{d}$.

Proof of Theorem 5.3. We first notice that, since (2.2) has not been modified, the results of Section 3 still hold.

Step 1. Existence and uniqueness of the approximate solution.

The existence and uniqueness of a solution to $((2.2)-(2.4),(5.13))$ is a consequence of the proof of Theorem 2.1 once we notice that, in this proof, $f_{K}=\int_{K} f(x) \mathrm{d} x$ can be replaced by any real number (and in particular $\left.\sum_{\sigma \in \mathcal{E}_{K}} \mathrm{~m}(\sigma) \mathbf{G}_{\sigma} \cdot \mathbf{n}_{K, \sigma}\right)$ without changing the reasoning.

Step 2. Estimates on the approximate solution.

In the following, we forget the indices $n$. To obtain estimates on the solution to $((2.2)-(2.4),(5.13))$, we make the manipulations at the beginning of the proof of Lemma 4.1 and we arrive at the equivalent of (4.16), which reads here

$$
\sum_{K \in \mathcal{M}} \mathrm{m}(K) a_{K}\left(\mathbf{v}_{K}\right) \cdot \mathbf{v}_{K}+\sum_{K \in \mathcal{M}} \sum_{\sigma \in \mathcal{E}_{K}} \nu_{K} \mathrm{~m}(K)\left(\left|F_{K, \sigma}\right|^{\frac{1}{p-1}}\right)^{p}=\sum_{K \in \mathcal{M}} u_{K} \sum_{\sigma \in \mathcal{E}_{K}} \mathrm{~m}(\sigma) \mathbf{G}_{\sigma} \cdot \mathbf{n}_{K, \sigma}
$$

Let $T_{8}$ be the right-hand side of this equation. Gathering by edges (notice that $\mathbf{G}_{\sigma} \cdot \mathbf{n}_{K, \sigma}=-\mathbf{G}_{\sigma} \cdot \mathbf{n}_{L, \sigma}$ if $\sigma=K \mid L)$, we have $T_{8}=\sum_{\sigma \in \mathcal{E}} \mathrm{m}(\sigma) \mathbf{G}_{\sigma} \cdot \mathbf{n}_{K, \sigma}\left(u_{K}-u_{L}\right.$ ) (we denote $\sigma=K \mid L$ if $\sigma \in \mathcal{E}_{\text {int }}$ and $u_{L}=0$ if 
$\left.\sigma \in \mathcal{E}_{\text {ext }} \cap \mathcal{E}_{K}\right)$. Hence, by (2.2) and defining $\mathbf{v}_{L}=\nu_{L}=F_{L, \sigma}=0$ if $\sigma \in \mathcal{E}_{\text {ext }} \cap \mathcal{E}_{K}$, we find

$$
\begin{aligned}
T_{8}= & -\sum_{\sigma \in \mathcal{E}} \mathrm{m}(\sigma) \mathbf{G}_{\sigma} \cdot \mathbf{n}_{K, \sigma}\left(\mathbf{v}_{K} \cdot\left(\mathbf{x}_{\sigma}-\mathbf{x}_{K}\right)+\mathbf{v}_{L} \cdot\left(\mathbf{x}_{L}-\mathbf{x}_{\sigma}\right)\right) \\
& -\sum_{\sigma \in \mathcal{E}} \mathrm{m}(\sigma) \mathbf{G}_{\sigma} \cdot \mathbf{n}_{K, \sigma}\left(\nu_{K} \mathrm{~m}(K)\left|F_{K, \sigma}\right|^{\frac{1}{p-1}-1} F_{K, \sigma}-\nu_{L} \mathrm{~m}(L)\left|F_{L, \sigma}\right|^{\frac{1}{p-1}-1} F_{L, \sigma}\right) \\
= & -\sum_{K \in \mathcal{M}} \mathbf{v}_{K} \cdot \sum_{\sigma \in \mathcal{E}_{K}} \mathrm{~m}(\sigma) \mathbf{G}_{\sigma} \cdot \mathbf{n}_{K, \sigma}\left(\mathbf{x}_{\sigma}-\mathbf{x}_{K}\right) \\
& -\sum_{K \in \mathcal{M}} \sum_{\sigma \in \mathcal{E}_{K}} \mathrm{~m}(\sigma) \mathbf{G}_{\sigma} \cdot \mathbf{n}_{K, \sigma} \nu_{K} \mathrm{~m}(K)\left|F_{K, \sigma}\right|^{\frac{1}{p-1}-1} F_{K, \sigma} \\
=: & T_{9}+T_{10} .
\end{aligned}
$$

We have, by (2.1),

$$
\begin{aligned}
\left|T_{9}\right| & \leq \omega_{d-1} \sum_{K \in \mathcal{M}} \sum_{\sigma \in \mathcal{E}_{K}} \operatorname{diam}(K)^{d}\left|\mathbf{v}_{K}\right|\left|\mathbf{G}_{\sigma}\right| \\
& \leq \frac{\omega_{d-1} \operatorname{regul}(\mathcal{D})}{\omega_{d}} \sum_{K \in \mathcal{M}} \sum_{\sigma \in \mathcal{E}_{K}} \mathrm{~m}(K)\left|\mathbf{v}_{K}\right|\left|\mathbf{G}_{\sigma}\right| \\
& \leq \frac{\omega_{d-1} \operatorname{regul}(\mathcal{D})}{\omega_{d}}\left(\sum_{K \in \mathcal{M}} \sum_{\sigma \in \mathcal{E}_{K}} \mathrm{~m}(K)\left|\mathbf{G}_{\sigma}\right|^{p^{\prime}}\right)^{\frac{1}{p^{\prime}}}\left(\sum_{K \in \mathcal{M}} \sum_{\sigma \in \mathcal{E}_{K}} \mathrm{~m}(K)\left|\mathbf{v}_{K}\right|^{p}\right)^{\frac{1}{p}}
\end{aligned}
$$

and

$$
\begin{aligned}
\left|T_{10}\right| \leq & \omega_{d-1} \sum_{K \in \mathcal{M}} \sum_{\sigma \in \mathcal{E}_{K}} \mathrm{~m}(K) \nu_{K} \operatorname{diam}(K)^{d-1}\left|F_{K, \sigma}\right|^{\frac{1}{p-1}}\left|\mathbf{G}_{\sigma}\right| \\
\leq & \omega_{d-1}\left(\sum_{K \in \mathcal{M}} \sum_{\sigma \in \mathcal{E}_{K}} \mathrm{~m}(K)\left|\mathbf{G}_{\sigma}\right|^{p^{\prime}}\right)^{\frac{1}{p^{\prime}}} \\
& \times\left(\sum_{K \in \mathcal{M}} \sum_{\sigma \in \mathcal{E}_{K}} \mathrm{~m}(K) \nu_{K}^{p} \operatorname{diam}(K)^{(d-1) p}\left|F_{K, \sigma}\right|^{\frac{p}{p-1}}\right)^{\frac{1}{p}} .
\end{aligned}
$$

Using Young inequalities, we deduce that for all $\varepsilon>0$ there exists $C_{24}$ depending on $\varepsilon$ but not on $\mathcal{D}$ (recall that $\operatorname{regul}(\mathcal{D})$ is bounded) such that

$$
\left|T_{8}\right| \leq C_{24} \sum_{K \in \mathcal{M}} \sum_{\sigma \in \mathcal{E}_{K}} \mathrm{~m}(K)\left|\mathbf{G}_{\sigma}\right|^{p^{\prime}}+\varepsilon\|\mathbf{v}\|_{L^{p}(\Omega)^{d}}^{p}+\varepsilon \sum_{K \in \mathcal{M}} \sum_{\sigma \in \mathcal{E}_{K}} \mathrm{~m}(K) \nu_{K}^{p} \operatorname{diam}(K)^{(d-1) p}\left|F_{K, \sigma}\right|^{\frac{p}{p-1}}
$$


Let us study the expression involving $\mathbf{G}_{\sigma}$ : by Jensen's inequality,

$$
\begin{aligned}
\sum_{K \in \mathcal{M}} \sum_{\sigma \in \mathcal{E}_{K}} \mathrm{~m}(K)\left|\mathbf{G}_{\sigma}\right|^{p^{\prime}} & \leq \sum_{K \in \mathcal{M}} \sum_{\sigma \in \mathcal{E}_{K}} \frac{\mathrm{m}(K)}{\mathrm{m}(K \cup L)} \int_{K \cup L}|\mathbf{G}(x)|^{p^{\prime}} \mathrm{d} x \\
& \leq \sum_{K \in \mathcal{M}} \sum_{\sigma \in \mathcal{E}_{K}}\left(\int_{K}|\mathbf{G}(x)|^{p^{\prime}} \mathrm{d} x+\int_{L}|\mathbf{G}(x)|^{p^{\prime}} \mathrm{d} x\right) \\
& \leq 2 \operatorname{regul}(\mathcal{D}) \sum_{K \in \mathcal{M}} \int_{K}|\mathbf{G}(x)|^{p^{\prime}} \mathrm{d} x \\
& \leq 2 \operatorname{regul}(\mathcal{D})\|\mathbf{G}\|_{L^{p^{\prime}}(\Omega)^{d}}^{p^{\prime}}
\end{aligned}
$$

(we have taken $L$ neighbor of $K$ such that $\sigma=K \mid L$, or $L=\emptyset$ if $\sigma \in \mathcal{E}_{\text {ext }} \cap \mathcal{E}_{K}$ ). We deduce that there exists $C_{25}$ depending on $\varepsilon$ and $\mathbf{G}$, but not on $\mathcal{D}$, such that

$$
\left|T_{8}\right| \leq C_{25}+\varepsilon|| \mathbf{v} \|_{L^{p}(\Omega)^{d}}^{p}+\varepsilon \sum_{K \in \mathcal{M}} \sum_{\sigma \in \mathcal{E}_{K}} \mathrm{~m}(K) \nu_{K}^{p} \operatorname{diam}(K)^{(d-1) p}\left|F_{K, \sigma}\right|^{\frac{p}{p-1}} .
$$

Injected in (5.14), this inequality leads to (4.17) and we can conclude, as in the proof of Lemma 4.1, that $\|\mathbf{v}\|_{L^{p}(\Omega)^{d}}$ and $\sum_{K \in \mathcal{M}} \sum_{\sigma \in \mathcal{E}_{K}} \nu_{K}\left(\left|F_{K, \sigma}\right|^{\frac{1}{p-1}}\right)^{p} \mathrm{~m}(K)$ stay bounded as $\operatorname{size}(\mathcal{D}) \rightarrow 0$.

Step 3. Convergence of the approximate solution.

Thanks to the preceding estimates, we can reason as in the proof of Theorem 2.2 and we arrive at (4.19). The convergence (4.20) is obtained as before, and it remains to study the limits of $\sum_{K \in \mathcal{M}} \mathrm{m}(K) a_{K}\left(\mathbf{v}_{K}\right) \cdot[\nabla \varphi]_{K}$ and $\sum_{K \in \mathcal{M}} \mathrm{m}(K) a_{K}\left(\mathbf{v}_{K}\right) \cdot \mathbf{v}_{K}$.

Let us first consider the second expression. By (5.14), we have

$$
\sum_{K \in \mathcal{M}} \mathrm{m}(K) a_{K}\left(\mathbf{v}_{K}\right) \cdot \mathbf{v}_{K} \leq T_{8}=T_{9}+T_{10}
$$

Inequalities (5.15) and (5.16) show that $\left|T_{10}\right| \leq C_{26} M_{p}(\mathcal{D}, \nu, F)$ where $C_{26}$ does not depend on $\mathcal{D}$. But $M_{p}(\mathcal{D}, \nu, F) \rightarrow 0$ as $\operatorname{size}(\mathcal{D}) \rightarrow 0$ (see $(4.18)$ ), and thus $T_{10} \rightarrow 0$ as $\operatorname{size}(\mathcal{D}) \rightarrow 0$.

Let $\varepsilon>0$ and $\widetilde{\mathbf{G}} \in C_{c}^{\infty}(\Omega)^{d}$ such that $\|\mathbf{G}-\widetilde{\mathbf{G}}\|_{L^{p^{\prime}}(\Omega)^{d}} \leq \varepsilon$. Let $\widetilde{\mathbf{G}}_{\sigma}$ be defined as $\mathbf{G}_{\sigma}$ but using $\widetilde{\mathbf{G}}$ instead of $\mathbf{G}$, and let

$$
T_{11}=-\sum_{K \in \mathcal{M}} \mathbf{v}_{K} \cdot \sum_{\sigma \in \mathcal{E}_{K}} \mathrm{~m}(\sigma) \widetilde{\mathbf{G}}_{\sigma} \cdot \mathbf{n}_{K, \sigma}\left(\mathbf{x}_{\sigma}-\mathbf{x}_{K}\right)
$$

(i.e. $T_{9}$ in which $\mathbf{G}_{\sigma}$ has been replaced by $\widetilde{\mathbf{G}}_{\sigma}$ ). We have, by (2.1),

$$
\begin{aligned}
\left|T_{9}-T_{11}\right| & \leq \omega_{d-1} \sum_{K \in \mathcal{M}} \sum_{\sigma \in \mathcal{E}_{K}} \operatorname{diam}(K)^{d}\left|\mathbf{v}_{K}\right|\left|\mathbf{G}_{\sigma}-\widetilde{\mathbf{G}}_{\sigma}\right| \\
& \leq \frac{\omega_{d-1} \operatorname{regul}(\mathcal{D})}{\omega_{d}} \sum_{K \in \mathcal{M}} \sum_{\sigma \in \mathcal{E}_{K}} \mathrm{~m}(K)\left|\mathbf{v}_{K}\right|\left|\mathbf{G}_{\sigma}-\widetilde{\mathbf{G}}_{\sigma}\right| \\
& \leq \frac{\omega_{d-1} \operatorname{regul}(\mathcal{D})}{\omega_{d}}\left(\sum_{K \in \mathcal{M}} \sum_{\sigma \in \mathcal{E}_{K}} \mathrm{~m}(K)\left|\mathbf{v}_{K}\right|^{p}\right)^{\frac{1}{p}}\left(\sum_{K \in \mathcal{M}} \sum_{\sigma \in \mathcal{E}_{K}} \mathrm{~m}(K)\left|\mathbf{G}_{\sigma}-\widetilde{\mathbf{G}}_{\sigma}\right|^{p^{\prime}}\right)^{\frac{1}{p^{\prime}}} \\
& \leq \frac{\omega_{d-1} \operatorname{regul}(\mathcal{D})}{\omega_{d}} \operatorname{regul}(\mathcal{D})^{\frac{1}{p}}|| \mathbf{v} \|_{L^{p}(\Omega)^{d}}\left(\sum_{K \in \mathcal{M}} \sum_{\sigma \in \mathcal{E}_{K}} \mathrm{~m}(K)\left|\mathbf{G}_{\sigma}-\widetilde{\mathbf{G}}_{\sigma}\right|^{p^{\prime}}\right)^{\frac{1}{p^{\prime}}}
\end{aligned}
$$


The same estimates that lead to (5.16), but applied to $\mathbf{G}-\widetilde{\mathbf{G}}$ instead of $\mathbf{G}$, show that the last term of this right-hand side is bounded by $(2 \operatorname{regul}(\mathcal{D}))^{1 / p^{\prime}}\|\mathbf{G}-\widetilde{\mathbf{G}}\|_{L^{p^{\prime}}(\Omega)^{d}}$. Hence,

$$
\left|T_{9}-T_{11}\right| \leq C_{27} \varepsilon
$$

where $C_{27}$ does not depend on $\varepsilon$ or $\mathcal{D}$ (recall that $\mathbf{v}$ is bounded in $\left.L^{p}(\Omega)^{d}\right)$. Let $\widetilde{\mathbf{G}}_{K}=\frac{1}{\mathrm{~m}(K)} \int_{K} \widetilde{\mathbf{G}}(x) \mathrm{d} x$. Since $\widetilde{\mathbf{G}}$ is regular we have, for all $K \in \mathcal{M}$, by Lemma 8.2 ,

$$
\begin{aligned}
\sum_{\sigma \in \mathcal{E}_{K}} \mathrm{~m}(\sigma) \widetilde{\mathbf{G}}_{\sigma} \cdot \mathbf{n}_{K, \sigma}\left(\mathbf{x}_{\sigma}-\mathbf{x}_{K}\right) & =\sum_{\sigma \in \mathcal{E}_{K}} \mathrm{~m}(\sigma) \widetilde{\mathbf{G}}_{K} \cdot \mathbf{n}_{K, \sigma}\left(\mathbf{x}_{\sigma}-\mathbf{x}_{K}\right)+\sum_{\sigma \in \mathcal{E}_{K}} \mathrm{~m}(\sigma) \mathbf{R}_{K, \sigma} \cdot \mathbf{n}_{K, \sigma}\left(\mathbf{x}_{\sigma}-\mathbf{x}_{K}\right) \\
& =\mathrm{m}(K) \widetilde{\mathbf{G}}_{K}+\sum_{\sigma \in \mathcal{E}_{K}} \mathrm{~m}(\sigma) \mathbf{R}_{K, \sigma} \cdot \mathbf{n}_{K, \sigma}\left(\mathbf{x}_{\sigma}-\mathbf{x}_{K}\right)
\end{aligned}
$$

where $\left|\mathbf{R}_{K, \sigma}\right| \leq C_{28}(\operatorname{diam}(K)+\operatorname{diam}(L)) \leq 2 C_{28} \operatorname{size}(\mathcal{D})$ with $C_{28}$ depending on $\widetilde{\mathbf{G}}$ but not on $\mathcal{D}$. Hence,

$$
\begin{aligned}
& \left|T_{11}+\sum_{K \in \mathcal{M}} \operatorname{m}(K) \mathbf{v}_{K} \cdot \widetilde{\mathbf{G}}_{K}\right| \\
& \quad \leq 2 C_{28} \operatorname{size}(\mathcal{D}) \omega_{d-1} \sum_{K \in \mathcal{M}} \sum_{\sigma \in \mathcal{E}_{K}} \operatorname{diam}(K)^{d}\left|\mathbf{v}_{K}\right| \\
& \quad \leq 2 C_{28} \operatorname{size}(\mathcal{D}) \omega_{d-1} \operatorname{regul}(\mathcal{D}) \frac{\operatorname{regul}(\mathcal{D})}{\omega_{d}}\|\mathbf{v}\|_{L^{1}(\Omega)^{d}} \rightarrow 0 \quad \operatorname{as~size}(\mathcal{D}) \rightarrow 0
\end{aligned}
$$

But $\sum_{K \in \mathcal{M}} \mathrm{m}(K) \mathbf{v}_{K} \cdot \widetilde{\mathbf{G}}_{K}=\int_{\Omega} \mathbf{v}(x) \cdot \widetilde{\mathbf{G}}(x) \mathrm{d} x \rightarrow \int_{\Omega} \nabla \bar{u}(x) \cdot \widetilde{\mathbf{G}}(x) \mathrm{d} x$ as $\operatorname{size}(\mathcal{D}) \rightarrow 0$, since $\mathbf{v} \rightarrow \nabla \bar{u}$ weakly in $L^{p}(\Omega)^{d}$. Moreover,

$$
\left|\int_{\Omega} \nabla \bar{u}(x) \cdot \widetilde{\mathbf{G}}(x) \mathrm{d} x-\int_{\Omega} \nabla \bar{u}(x) \cdot \mathbf{G}(x) \mathrm{d} x\right| \leq\|\nabla \bar{u}\|_{L^{p}(\Omega)^{d}}\|\mathbf{G}-\widetilde{\mathbf{G}}\|_{L^{p^{\prime}}(\Omega)^{d}} \leq \varepsilon\|\nabla \bar{u}\|_{L^{p}(\Omega)^{d}} .
$$

Therefore, by (5.18) and (5.19),

$$
\limsup _{\operatorname{size}(\mathcal{D}) \rightarrow 0}\left|T_{9}+\int_{\Omega} \nabla \bar{u}(x) \cdot \mathbf{G}(x) \mathrm{d} x\right| \leq\left(C_{27}+\|\nabla \bar{u}\|_{L^{p}(\Omega)^{d}}\right) \varepsilon
$$

Since this is true for all $\varepsilon>0$ and since $C_{27}$ does not depend on $\varepsilon$, we deduce that $T_{9} \rightarrow-\int_{\Omega} \nabla \bar{u}(x) \cdot \mathbf{G}(x) \mathrm{d} x$ as $\operatorname{size}(\mathcal{D}) \rightarrow 0$. Recalling that $T_{10} \rightarrow 0$, we deduce from (5.17) that

$$
\limsup _{\operatorname{size}(\mathcal{D}) \rightarrow 0} \sum_{K \in \mathcal{M}} \mathrm{m}(K) a_{K}\left(\mathbf{v}_{K}\right) \cdot \mathbf{v}_{K} \leq-\int_{\Omega} \nabla \bar{u}(x) \cdot \mathbf{G}(x) \mathrm{d} x .
$$

To study the convergence of $\sum_{K \in \mathcal{M}} \mathrm{m}(K) a_{K}\left(\mathbf{v}_{K}\right) \cdot[\nabla \varphi]_{K}$, we make the manipulations that allowed to write (4.22); here, they give, using (5.13),

$$
\begin{aligned}
\sum_{K \in \mathcal{M}} \mathrm{m}(K) a_{K}\left(\mathbf{v}_{K}\right) \cdot[\nabla \varphi]_{K} & =\sum_{K \in \mathcal{M}} \varphi\left(\mathbf{x}_{K}\right) \sum_{\sigma \in \mathcal{E}_{K}} \mathrm{~m}(\sigma) \mathbf{G}_{\sigma} \cdot \mathbf{n}_{K, \sigma}+T_{7} \\
& =\sum_{\sigma \in \mathcal{E}} \mathrm{m}(\sigma) \mathbf{G}_{\sigma} \cdot \mathbf{n}_{K, \sigma}\left(\varphi\left(\mathbf{x}_{K}\right)-\varphi\left(\mathbf{x}_{L}\right)\right)+T_{7}
\end{aligned}
$$


(where $T_{7}$ is the same term as in the proof of Theorem 2.2 , and thus tends to 0 as $\operatorname{size}(\mathcal{D}) \rightarrow 0$, thanks to the estimate we have on the fluxes $\left.F_{K, \sigma}\right)$. We then re-use (4.21) to find

$$
\begin{aligned}
\sum_{K \in \mathcal{M}} \mathrm{m}(K) a_{K}\left(\mathbf{v}_{K}\right) \cdot[\nabla \varphi]_{K} & =-\sum_{\sigma \in \mathcal{E}} \mathrm{m}(\sigma) \mathbf{G}_{\sigma} \cdot \mathbf{n}_{K, \sigma}\left([\nabla \varphi]_{K} \cdot\left(\mathbf{x}_{\sigma}-\mathbf{x}_{K}\right)+[\nabla \varphi]_{L} \cdot\left(\mathbf{x}_{L}-\mathbf{x}_{\sigma}\right)\right)+T_{12}+T_{7} \\
& =-\sum_{K \in \mathcal{M}}[\nabla \varphi]_{K} \cdot \sum_{\sigma \in \mathcal{E}_{K}} \mathrm{~m}(\sigma) \mathbf{G}_{\sigma} \cdot \mathbf{n}_{K, \sigma}\left(\mathbf{x}_{\sigma}-\mathbf{x}_{K}\right)+T_{12}+T_{7}
\end{aligned}
$$

where $\left|T_{12}\right| \leq C_{29} \sum_{\sigma \in \mathcal{E}} \mathrm{m}(\sigma)\left|\mathbf{G}_{\sigma}\right|\left(\operatorname{diam}(K)^{2}+\operatorname{diam}(L)^{2}\right)$ with $C_{29}$ only depending on $\varphi$. Using the same techniques as in the convergence of $T_{9}$ above, it is easy to see that the first term of the right-hand side of (5.21) tends to $-\int_{\Omega} \nabla \varphi(x) \cdot \mathbf{G}(x) \mathrm{d} x$ as $\operatorname{size}(\mathcal{D}) \rightarrow 0$. Moreover,

$$
\begin{aligned}
\left|T_{12}\right| & \leq C_{29} \sum_{K \in \mathcal{M}} \operatorname{diam}(K)^{2} \sum_{\sigma \in \mathcal{E}_{K}} \mathrm{~m}(\sigma)\left|\mathbf{G}_{\sigma}\right| \\
& \leq C_{29} \omega_{d-1} \operatorname{size}(\mathcal{D}) \sum_{K \in \mathcal{M}} \sum_{\sigma \in \mathcal{E}_{K}} \operatorname{diam}(K)^{d}\left|\mathbf{G}_{\sigma}\right| \\
& \leq C_{29} \omega_{d-1} \operatorname{size}(\mathcal{D}) \frac{\operatorname{regul}(\mathcal{D})}{\omega_{d}} \sum_{K \in \mathcal{M}} \sum_{\sigma \in \mathcal{E}_{K}} \mathrm{~m}(K)\left|\mathbf{G}_{\sigma}\right| \\
& \leq C_{29} \omega_{d-1} \operatorname{size}(\mathcal{D}) \frac{\operatorname{regul}(\mathcal{D})}{\omega_{d}}\left(\sum_{K \in \mathcal{M}} \sum_{\sigma \in \mathcal{E}_{K}} \mathrm{~m}(K)\left|\mathbf{G}_{\sigma}\right|^{p^{\prime}}\right)^{\frac{1}{p^{\prime}}}(\operatorname{regul}(\mathcal{D}) \mathrm{m}(\Omega))^{\frac{1}{p}}
\end{aligned}
$$

and this right-hand side tends to 0 as $\operatorname{size}(\mathcal{D}) \rightarrow 0$, thanks to $(5.16)$. Hence, (5.21) shows that

$$
\sum_{K \in \mathcal{M}} \mathrm{m}(K) a_{K}\left(\mathbf{v}_{K}\right) \cdot[\nabla \varphi]_{K} \rightarrow-\int_{\Omega} \nabla \varphi(x) \cdot \mathbf{G}(x) \mathrm{d} x \quad \text { as } \operatorname{size}(\mathcal{D}) \rightarrow 0 .
$$

Thanks to this convergence, to (4.20) and to (5.20), we can take the limsup as size $(\mathcal{D}) \rightarrow 0$ of $(4.19)$ and we obtain

$$
0 \leq \int_{\Omega} a(x, \nabla \varphi(x)) \cdot(\nabla \varphi(x)-\nabla \bar{u}(x)) \mathrm{d} x+\int_{\Omega} \mathbf{G}(x) \cdot(\nabla \varphi(x)-\nabla \bar{u}(x)) \mathrm{d} x .
$$

The conclusion that $\bar{u}$ is a weak solution to (1.1) with $f=\operatorname{div}(\mathbf{G})$ and that $\mathbf{v}$ strongly converges to $\nabla \bar{u}$ then follows exactly as in the proof of Theorem 2.2 .

Remark 5.4. This handling of right-hand sides in $W^{-1, p^{\prime}}(\Omega)$ can be simultaneously conducted with all or part of the techniques in the preceding subsections.

Notice however that, to apply the non-penalized scheme to a right-hand side which belongs to $W^{-1, p^{\prime}}(\Omega)$, one needs a more precise estimate than (5.11) on the matrix $A_{K}$ : we can see [12], proof of Lemma 6.4 , that, in fact, the coefficients in the first column of $A_{K}^{-1}$ are of order 1 (and not $\operatorname{diam}(K)^{-1}$ ). Hence, when estimating $F_{K}$ by inverting (5.10) with $\int_{K} f(x) \mathrm{d} x$ replaced by $\sum_{\sigma \in \mathcal{E}_{K}} \mathrm{~m}(\sigma) \mathbf{G}_{\sigma} \cdot \mathbf{n}_{K, \sigma}$, this last term appears with coefficients of order 1 ; once we have factorized $\operatorname{diam}(K)^{-1}$, the coefficients of $\mathbf{G}_{\sigma}$ are of order $\operatorname{diam}(K)^{d} \sim \mathrm{m}(K)$, which is sufficient to obtain a good estimate on the fluxes (see (5.16)).

\section{Numerical RESUlts}

We present here some numerical results. The domain $\Omega=]-1,1{ }^{2}$ is discretized using triangular grids; each point $\mathbf{x}_{K}$ is randomly chosen inside its control volume $K$. The equation we study is the $p$-Laplacian: $-\operatorname{div}\left(|\nabla \bar{u}|^{p-2} \nabla \bar{u}\right)=f$, where the right-hand side $f$ is taken so that the exact solution is $\bar{u}(x, y)=\left(x^{2}-1\right)\left(y^{2}-1\right)$ 


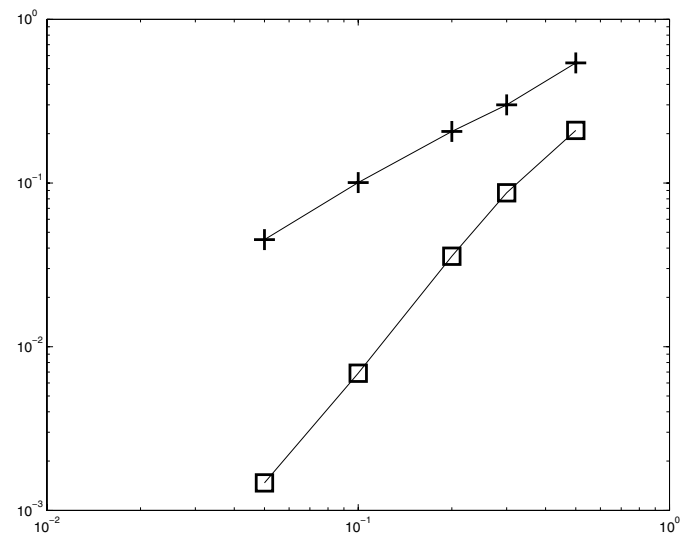

Figure 1. $p=1.5$. Relative errors $\frac{\|u-\bar{u}\|_{p}}{\|\bar{u}\|_{p}}$ (" $\square$ "; slope $\left.\approx 2.1\right)$ and $\frac{\|\mathbf{v}-\nabla \bar{u}\|_{p}}{\|\nabla \bar{u}\|_{p}}$ $("+"$; slope $\approx 1)$ versus the size of the discretization, in log-log scale.

(one can check that this right-hand side belongs to $L^{p^{\prime}}(\Omega)$ provided that $p>\sqrt{2}$ ). We are mainly interested in the way our scheme behaves with respect to $p$.

Remark 6.1. The non-linear system $((2.2)-(2.5))$, or its non-penalized version, is of size $(d+1) \operatorname{Card}(\mathcal{M})+\operatorname{Card}(\mathcal{E})$ (thanks to $(2.3)$, we can consider that there are as many fluxes as there are edges). This is quite big; however, if we compute its solution via a Newton method (which requires the resolution of a linearized version of this system), we can use the hybridization method of [12] to reduce the linear systems to solve, at each iteration of the Newton method, to systems of size $\operatorname{Card}\left(\mathcal{E}_{\text {int }}\right)$.

Notice that, strictly speaking, the function $\mathcal{G}$ defining the system $((2.2)-(2.5))$ is not regular if $p>2$ (because of the term $\left.\left|F_{K, \sigma}\right|^{\frac{1}{p-1}-1} F_{K, \sigma}\right)$, which could be considered as an issue for its linearization in the Newton method. However, the set where $\mathcal{G}$ is not regular is very thin (from a numerical point of view, there is nearly no chance to fall in this set while applying the Newton algorithm) and, above all, the existence of a solution to the scheme has been obtained by a topological degree argument; this solution is therefore stable and, if one wants to be sure to apply the Newton method on a regular function, it is possible to consider an obvious regular approximation of $\mathcal{G}$ (with the same structure as $\mathcal{G}$ and, in particular, the same hybridation properties) and to search for a zero of this regularization, hence obtaining a good approximation of the zero of $\mathcal{G}$. However, in practical situations, this is not really necessary.

Remark 6.2. The grids we consider here are simplicial, so that the non-penalized version of the scheme can be applied. When using other grids, the question arises as how to choose the penalization parameters $\nu_{0}$ and $\beta$. The numerical tests on non-simplicial grids such as the ones in [12] give similar (and sometimes even better) results to the ones we present below and show very little dependence on the choice of the penalization parameters "within a reasonable range": any $\nu_{0} \in\left[10^{-3}, 10^{-10}\right]$ and $\left.\beta \in\right]-p^{\prime}(d-1),-p^{\prime}(d-2)[$ provide good numerical results. Only very small values of $\nu_{0}$ (below $10^{-11}$ ) should be avoided since, in some cases, they can give rise to numerical issues in the Newton algorithm; large values of $\nu_{0}$ are not really a problem, except that they demand very thin grids in order to obtain correct approximations of the solution.

Figure 1 shows the relative errors $\frac{\|u-\bar{u}\|_{p}}{\|\bar{u}\|_{p}}$ and $\frac{\|\mathbf{v}-\nabla \bar{u}\|_{p}}{\|\nabla \bar{u}\|_{p}}$ versus the size of the discretization, in log-log scale and for $p=1.5$. The rates of convergence are 2.1 for $u$ and 1 for $\mathbf{v}$ (the results for $p=2$, not shown here, exhibit the same slopes and, in fact, the graphs of the errors for $p=1.5$ and $p=2$ can nearly be superimposed).

We have also run experiments for $p=1.1$ ( $f$ then does not belong to $L^{p^{\prime}}(\Omega)$, but its singularities are isolated so we have still used (2.5) instead of (5.13)): the results are quite similar, and even better for $u$ (the rates of convergence for $u$ and $\mathbf{v}$ are respectively 2.5 and 1). All these tests show something that often appears 


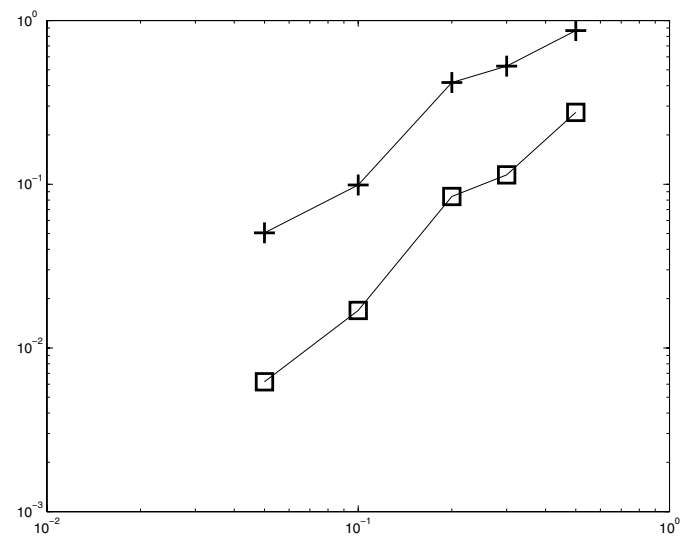

FiguRE 2. $p=4$. Relative errors $\frac{\|u-\bar{u}\|_{p}}{\|\mid \bar{u}\|_{p}}$ (" $\square$ "; slope $\approx 1.6$ ) and $\frac{\|\mathbf{v}-\nabla \bar{u}\|_{p}}{\|\nabla \bar{u}\|_{p}}$ $("+"$ " slope $\approx 1.2$ ) versus the size of the discretization, in log-log scale.

when discretizing PDEs on regular meshes: a super-convergence of the approximate solution with respect to its gradient; notice however that our meshes are not regular, since the $\mathbf{x}_{K}$ have been randomly chosen and are not located at the center of each control volume.

The results for $p=4$ are presented in Figure 2. The rate of convergence for $u$ (roughly 1.6) is worst than in the cases $p \leq 2$; curiously enough, the rate for $\mathbf{v}$ (roughly 1.2) seems slightly better than with the preceding choices of $p$, but this does not last: as $p$ increases, we notice that the rates of convergence for both $u$ and $\mathbf{v}$ diminish (for $p=6$, these rates are respectively equal to 1 and 0.3 ). This phenomenon is probably due to the fact that, if $p>2$, the factor $|\nabla \bar{u}|^{p-2}$ vanishes as $\nabla \bar{u} \rightarrow 0$ (in which case $-\operatorname{div}\left(|\nabla \bar{u}|^{p-2} \nabla \bar{u}\right.$ ) is a degenerate elliptic operator), and it can be compared with the lack of regularity of the solution for large $p$ : if $p>2$, there exists right-hand sides in $L^{p^{\prime}}(\Omega)$ such that the solution does not belong to $W^{2, p}(\Omega)$ (this never happens if $p \leq 2$, see $[5])$.

In [8] and [5], some theoretical error estimates are given for the approximation by the Finite Element method of the $p$-Laplacian equation in dimension $d=2$; these estimates are of order $p / 2$ (if $p \leq 2$ ) or $2 / p$ (if $p \geq 2$ ) in the $W^{1, p}$ norm. The preceding numerical results show that our scheme has a better behavior in the vast majority of cases: for $p=1.5$, the gradients converge with order 1 in our scheme and (theoretically) with order 0.75 in the Finite Element scheme, and for $p=4$ (respectively $p=6$ ) our convergence is of order 1.2 (respectively 0.3 ) whereas the order in [8] is 0.5 (respectively 0.33 ).

Reference [21] studies the Finite Element approximation of a problem similar to (1.1) with $p=1.5$, and they observe a convergence of order 0.95 , which is the same magnitude as the order 1 our scheme provides in this case. In [4], numerical results are given for another Finite Volume scheme for anisotropic and nonlinear elliptic equations in dimension $d=2$. For anisotropic linear equations, the rate of convergence is 0.5 in $H^{1}$ norm, which is less that our scheme: as explained in [12], we observe in general an order of convergence 1 in this situation. They also give orders of convergence for nonlinear equations with different values of $p$ : for example, with $p=4$ they show orders 1.83 and 0.84 for the approximations of the function and its gradient (we respectively have 1.6 and 1.2) and, with $p=6$, the orders are 1.65 and 0.65 (versus 1 and 0.3 for our scheme). Notice however that these comparisons become sketchy since the operator is not exactly the $p$-Laplacian, and the exact solution and meshes do not coincide with the ones we have taken.

\section{Conclusion}

To conclude, we constructed Finite Volume schemes for nonlinear elliptic equations of the Leray-Lions type (the $p$-Laplacian being the canonical example of these equations). 
We defined spaces of functions associated with these schemes and we proved general properties on these spaces (Poincaré's inequality, compactness property), which allowed us to make the theoretical study of the schemes. We showed the strong convergence of the approximate solutions and the approximate gradients towards the solution of the PDE and its gradient, even in the case of a non-monotone operator or for right-hand sides with little regularity.

The convergences hold for very general meshes (even unstructured or non-admissible ones, with respect to the definition of [15]), with minimal assumptions; the proofs needed very few geometric considerations, which allowed them to work in any space dimension and makes of this scheme a good candidate to handle complex geometries and/or strongly coupled equations (as shown in [7]), all the more as the approximate gradients strongly converge. We provided numerical results which show good performances for the scheme (in most situations comparable to or better than other schemes).

\section{Appendix}

Lemma 8.1. Let $r \in\left[1, \infty\left[\right.\right.$ and $K$ be a non empty open polygonal convex set in $\mathbb{R}^{d}$ such that, for some $\alpha>0$, there exists a ball of radius $\alpha \operatorname{diam}(K)$ contained in $K$. Let $E$ be an affine hyperplane of $\mathbb{R}^{d}$ and $\sigma$ be a nonempty open subset of $E$ contained in $\partial K \cap E$. Then there exists $C_{30}$ only depending on $r, d$ and $\alpha$ such that, for all $v \in W^{1, r}(K)$,

$$
\left|\frac{1}{\mathrm{~m}(\sigma)} \int_{\sigma} v \mathrm{~d} \gamma\right|^{r} \leq \frac{C_{30} \operatorname{diam}(K)^{r}}{\mathrm{~m}(\sigma) \operatorname{diam}(K)} \int_{K}|\nabla v(x)|^{r} \mathrm{~d} x+\frac{C_{30}}{\mathrm{~m}(\sigma) \operatorname{diam}(K)} \int_{K}|v(x)|^{r} \mathrm{~d} x .
$$

Proof of Lemma 8.1. Since $K$ is convex, the regular functions are dense in $W^{1, r}(K)$ and it suffices to prove the result for $v \in C^{1}\left(\mathbb{R}^{d}\right)$. Let $\mathbf{p}_{K}$ be the center of a ball of radius $\alpha \operatorname{diam}(K)$ contained in $K$, and denote $\triangle_{K, \sigma}$ the convex hull of $\mathbf{p}_{K}$ and $\sigma$. The last inequality in the proof of Lemma 6.2 in [12] reads

$$
\left|\frac{1}{\mathrm{~m}\left(\triangle_{K, \sigma}\right)} \int_{\triangle_{K, \sigma}} v(x) \mathrm{d} x-\frac{1}{\mathrm{~m}(\sigma)} \int_{\sigma} v \mathrm{~d} \gamma\right| \leq \frac{C_{31} \operatorname{dist}\left(\mathbf{p}_{K}, E\right)}{\mathrm{m}\left(\triangle_{K, \sigma}\right)} \int_{\triangle_{K, \sigma}}|\nabla v(x)| \mathrm{d} x
$$

where $C_{31}$ only depends on $\alpha$. Hence, $\operatorname{since} \operatorname{dist}\left(\mathbf{p}_{K}, E\right) \leq \operatorname{dist}\left(\mathbf{p}_{K}, \sigma\right) \leq \operatorname{diam}(K)$,

$$
\left|\frac{1}{\mathrm{~m}(\sigma)} \int_{\sigma} v \mathrm{~d} \gamma\right| \leq \frac{C_{31} \operatorname{diam}(K)}{\mathrm{m}\left(\triangle_{K, \sigma}\right)} \int_{\triangle_{K, \sigma}}|\nabla v(x)| \mathrm{d} x+\frac{1}{\mathrm{~m}\left(\triangle_{K, \sigma}\right)} \int_{\triangle_{K, \sigma}}|v(x)| \mathrm{d} x .
$$

We have $\mathrm{m}\left(\triangle_{K, \sigma}\right)=\frac{\mathrm{m}(\sigma) \operatorname{dist}\left(\mathbf{p}_{K}, E\right)}{d}$, and Jensen's inequality therefore gives

$$
\begin{aligned}
\left|\frac{1}{\mathrm{~m}(\sigma)} \int_{\sigma} v \mathrm{~d} \gamma\right|^{r} & \leq \frac{2^{r-1} C_{31}^{r} \operatorname{diam}(K)^{r}}{\mathrm{~m}\left(\triangle_{K, \sigma}\right)} \int_{\triangle_{K, \sigma}}|\nabla v(x)|^{r} \mathrm{~d} x+\frac{2^{r-1}}{\mathrm{~m}\left(\triangle_{K, \sigma}\right)} \int_{\triangle_{K, \sigma}}|v(x)|^{r} \mathrm{~d} x \\
& \leq \frac{2^{r-1} d C_{31}^{r} \operatorname{diam}(K)^{r}}{\mathrm{~m}(\sigma) \operatorname{dist}\left(\mathbf{p}_{K}, E\right)} \int_{K}|\nabla v(x)|^{r} \mathrm{~d} x+\frac{2^{r-1} d}{\mathrm{~m}(\sigma) \operatorname{dist}\left(\mathbf{p}_{K}, E\right)} \int_{K}|v(x)|^{r} \mathrm{~d} x .
\end{aligned}
$$

Since $K$ is convex and $\partial K \cap E$ contains a non-empty open subset of $E, K$ is on one side of $E$; hence, the ball of center $\mathbf{p}_{K}$ and radius $\alpha \operatorname{diam}(K)$ is also on one side of $E$ and $\operatorname{dist}\left(\mathbf{p}_{K}, E\right) \geq \alpha \operatorname{diam}(K)$. The preceding estimate then concludes the proof.

Lemma 8.2. Let $K$ be a non empty open convex polygonal set in $\mathbb{R}^{d}$. For $\sigma \in \mathcal{E}_{K}$ (the edges of $K$, in the sense given in Def. 2.1), we let $\mathbf{x}_{\sigma}$ be the center of gravity of $\sigma$; we also denote $\mathbf{n}_{K, \sigma}$ the unit normal to $\sigma$ outward 
to $K$. Then, for all vector $\mathbf{e} \in \mathbb{R}^{d}$ and for all point $\mathbf{x}_{K} \in K$, we have

$$
\mathrm{m}(K) \mathbf{e}=\sum_{\sigma \in \mathcal{E}_{K}} \mathrm{~m}(\sigma) \mathbf{e} \cdot \mathbf{n}_{K, \sigma}\left(\mathbf{x}_{\sigma}-\mathbf{x}_{K}\right) .
$$

The proof of Lemma 8.2 is a straightforward application of Stokes' formula on the function $x \rightarrow\left(x^{i}-\mathbf{x}_{K}^{i}\right) \mathbf{e}$ (where the superscript $i$ denotes the $i$-th component). It can be found in [12].

Lemma 8.3. Assume (1.2) and (1.5). Let $\psi \in C_{c}(\Omega)^{d}$. For $\mathcal{D}$ an admissible discretization of $\Omega$, we define $a_{\mathcal{D}}: \Omega \times \mathbb{R}^{d} \rightarrow \mathbb{R}^{d}$ and $\psi_{\mathcal{D}}: \Omega \rightarrow \mathbb{R}^{d}$ by

$$
\begin{gathered}
\text { for all } K \in \mathcal{M} \text { and all } \xi \in \mathbb{R}^{d}, a_{\mathcal{D}}(\cdot, \xi)=a_{K}(\xi)=\frac{1}{\mathrm{~m}(K)} \int_{K} a(x, \xi) \mathrm{d} x \text { on } K, \\
\qquad \text { for all } K \in \mathcal{M}, \psi_{\mathcal{D}}=\psi_{K}:=\frac{1}{\mathrm{~m}(K)} \int_{K} \psi(x) \mathrm{d} x \text { on } K .
\end{gathered}
$$

Then $a_{\mathcal{D}}\left(\cdot, \psi_{\mathcal{D}}\right) \rightarrow a(\cdot, \psi)$ strongly in $L^{p^{\prime}}(\Omega)^{d}$ as $\operatorname{size}(\mathcal{D}) \rightarrow 0$.

Proof of Lemma 8.3. Let $(a(\cdot, \psi))_{\mathcal{D}}: \Omega \rightarrow \mathbb{R}^{d}$ be the piecewise constant function equal, on each control volume $K \in \mathcal{M}$, to the mean value of $a(\cdot, \psi)$ on $K$. We first compare $a_{\mathcal{D}}\left(\cdot, \psi_{\mathcal{D}}\right)$ with $(a(\cdot, \psi))_{\mathcal{D}}$; by Jensen's inequality, we have

$$
\begin{aligned}
\int_{\Omega} \mid a_{\mathcal{D}}\left(x, \psi_{\mathcal{D}}(x)\right)- & \left.(a(\cdot, \psi))_{\mathcal{D}}(x)\right|^{p^{\prime}} \mathrm{d} x \\
& =\sum_{K \in \mathcal{M}} \int_{K}\left|a_{\mathcal{D}}\left(x, \psi_{\mathcal{D}}(x)\right)-(a(\cdot, \psi))_{\mathcal{D}}(x)\right|^{p^{\prime}} \mathrm{d} x \\
& =\sum_{K \in \mathcal{M}} \mathrm{m}(K)\left|\frac{1}{\mathrm{~m}(K)} \int_{K} a\left(y, \psi_{K}\right) \mathrm{d} y-\frac{1}{\mathrm{~m}(K)} \int_{K} a(y, \psi(y)) \mathrm{d} y\right|^{p^{\prime}} \\
& \leq \sum_{K \in \mathcal{M}} \int_{K}\left|a\left(y, \psi_{K}\right)-a(y, \psi(y))\right|^{p^{\prime}} \mathrm{d} y \\
& =\int_{\Omega}\left|a\left(y, \psi_{\mathcal{D}}(y)\right)-a(y, \psi(y))\right|^{p^{\prime}} \mathrm{d} y .
\end{aligned}
$$

For all $y \in \Omega$, the function $a(y, \cdot)$ is continuous on $\mathbb{R}^{d}$ and $\psi_{\mathcal{D}}(y) \rightarrow \psi(y)$ as $\operatorname{size}(\mathcal{D}) \rightarrow 0$ (because $\psi$ is continuous); hence, $\left|a\left(y, \psi_{\mathcal{D}}(y)\right)-a(y, \psi(y))\right|^{p^{\prime}} \rightarrow 0$ as $\operatorname{size}(\mathcal{D}) \rightarrow 0$. Moreover, by $(1.5)$,

$$
\begin{aligned}
\left|a\left(y, \psi_{\mathcal{D}}(y)\right)-a(y, \psi(y))\right|^{p^{\prime}} & \leq 2^{p^{\prime}-1}\left(2^{p^{\prime}-1} b(y)^{p^{\prime}}+2^{p^{\prime}-1} \Lambda^{p^{\prime}}\left\|\psi_{\mathcal{D}}\right\|_{\infty}^{p}+2^{p^{\prime}-1} b(y)^{p^{\prime}}+2^{p^{\prime}-1} \Lambda^{p^{\prime}}\|\psi\|_{\infty}^{p}\right) \\
& \leq 2^{2\left(p^{\prime}-1\right)+1} b(y)^{p^{\prime}}+2^{2\left(p^{\prime}-1\right)+1} \Lambda^{p^{\prime}}\|\psi\|_{\infty}^{p} .
\end{aligned}
$$

Since $b \in L^{p^{\prime}}(\Omega)$, the dominated convergence theorem then implies that the right-hand side of (8.1) tends to 0 as $\operatorname{size}(\mathcal{D}) \rightarrow 0$, which shows that $a_{\mathcal{D}}\left(\cdot, \psi_{\mathcal{D}}\right)-(a(\cdot, \psi))_{\mathcal{D}} \rightarrow 0$ in $L^{p^{\prime}}(\Omega)^{d}$.

As $a(\cdot, \psi) \in L^{p^{\prime}}(\Omega)^{d}$ (see $\left.(1.5)\right)$, it is classical that $(a(\cdot, \psi))_{\mathcal{D}} \rightarrow a(\cdot, \psi)$ in $L^{p^{\prime}}(\Omega)^{d}$ as $\operatorname{size}(\mathcal{D}) \rightarrow 0$, and the proof is therefore concluded.

The following lemma is a very easy result, quite classical in the study of non-linear elliptic equations.

Lemma 8.4. Let $\left(f_{n}\right)_{n \geq 1}$ be a sequence of nonnegative functions which converge a.e. on $\Omega$ to $f \in L^{1}(\Omega)$. If $\int_{\Omega} f_{n}(x) \mathrm{d} x \rightarrow \int_{\Omega} f(x) \mathrm{d} x$ as $n \rightarrow \infty$, then $f_{n} \rightarrow f$ in $L^{1}(\Omega)$. 
Proof of Lemma 8.4. We first notice that $\left(f-f_{n}\right)^{+} \leq f$, since $f_{n} \geq 0$. Hence, since $f$ is integrable, the dominated convergence theorem gives $\int_{\Omega}\left(f(x)-f_{n}(x)\right)^{+} \mathrm{d} x \rightarrow 0$ as $n \rightarrow \infty$. We then write $\int_{\Omega}\left(f(x)-f_{n}(x)\right) \mathrm{d} x=$ $\int_{\Omega}\left(f(x)-f_{n}(x)\right)^{+} \mathrm{d} x-\int_{\Omega}\left(f(x)-f_{n}(x)\right)^{-} \mathrm{d} x$ so that

$$
\begin{aligned}
\int_{\Omega}\left|f(x)-f_{n}(x)\right| \mathrm{d} x & =\int_{\Omega}\left(f(x)-f_{n}(x)\right)^{+} \mathrm{d} x+\int_{\Omega}\left(f(x)-f_{n}(x)\right)^{-} \mathrm{d} x \\
& =2 \int_{\Omega}\left(f(x)-f_{n}(x)\right)^{+} \mathrm{d} x-\int_{\Omega}\left(f(x)-f_{n}(x)\right) \mathrm{d} x
\end{aligned}
$$

and the proof is concluded.

\section{REFERENCES}

[1] S. Agmon, A. Douglis and L. Niremberg, Estimates near the boundary for solutions of elliptic partial differential equations satisfying general boundary conditions, Part I and Part II. Comm. Pure. Appl. Math. 12 (1959) 623-727 and 17 (1964) 35-92.

[2] B. Andreianov, F. Boyer and F. Hubert, Finite-volume schemes for the $p$-laplacian on cartesian meshes. ESAIM: M2AN 38 (2004) 931-960.

[3] B. Andreianov, F. Boyer and F. Hubert, Besov regularity and new error estimates for finite volume approximation of the p-Laplacian. Numer. Math. 100 (2005) 565-592.

[4] B. Andreianov, F. Boyer and F. Hubert, Discrete duality finite volume schemes for Leray-Lions type elliptic problems on general 2D meshes. Numer. Methods Partial Differ. Equ. 23 (2007) 145-195.

[5] J.W. Barrett and W.B. Liu, A remark on the regularity of the solutions of the $p$-Laplacian and its application to the finite element approximation. J. Math. Anal. Appl. 178 (1993) 470-487.

[6] L. Boccardo, T. Gallouët and F. Murat, Unicité de la solution de certaines équations elliptiques non linéaires. C.R. Acad. Sci. Paris 315 (1992) 1159-1164.

[7] C. Chainais and J. Droniou, Convergence analysis of a mixed finite volume scheme for an elliptic-parabolic system modeling miscible fluid flows in porous media, submitted. Available at http://hal.ccsd.cnrs.fr/ccsd-00022910.

[8] S. Chow, Finite element error estimates for non-linear elliptic equations of monotone type. Numer. Math. 54 (1989) $373-393$.

[9] Y. Coudiere, J.-P. Vila and P. Villedieu, Convergence rate of a finite volume scheme for a two dimensional convection-diffusion problem. ESAIM: M2AN 33 (1999) 493-516.

[10] K. Deimling, Nonlinear functional analysis. Springer (1985).

[11] J.I. Diaz and F. de Thelin, On a nonlinear parabolic problem arising in some models related to turbulent flows. SIAM J. Math. Anal. 25 (1994) 1085-1111.

[12] J. Droniou and R. Eymard, A mixed finite volume scheme for anisotropic diffusion problems on any grid. Num. Math. 105 (2006) 35-71.

[13] J. Droniou and R. Eymard, Study of the mixed finite volume method for Stokes and Navier-Stokes equations, submitted. Available at http://hal.archives-ouvertes.fr/hal-00110911.

[14] J. Droniou and T. Gallouët, Finite volume methods for convection-diffusion equations with right-hand side in $H^{-1}$. ESAIM: M2AN 36 (2002) 705-724.

[15] R. Eymard, T. Gallouët and R. Herbin, Finite Volume Methods, Handbook of Numerical Analysis, P.G. Ciarlet and J.L. Lions Eds., Vol. VII, 713-1020 (North Holland).

[16] M. Feistauer and A. Ženíšek, Finite element solution of nonlinear elliptic problems. Numer. Math. 50 (1987) 451-475.

[17] M. Feistauer and A. Ženíšek, Compactness method in the finite element theory of nonlinear elliptic problems. Numer. Math. 52 (1988) 147-163.

[18] M. Feistauer and V. Sobotíková, Finite element approximation of nonlinear elliptic problems with discontinuous coefficients. RAIRO Modél. Math. Anal. Numér. 24 (1990) 457-500.

[19] J.M. Fiard and R. Herbin, Comparison between finite volume finite element methods for the numerical simulation of an elliptic problem arising in electrochemical engineering. Comput. Meth. Appl. Mech. Engin. 115 (1994) 315-338.

[20] R. Glowinski, Numerical methods for nonlinear variational problems. Springer (1984).

[21] R. Glowinski and J. Rappaz, Approximation of a nonlinear elliptic problem arising in a non-newtonian fluid flow model in glaciology. ESAIM: M2AN 37 (2003) 175-186.

[22] J. Leray and J.L. Lions, Quelques résultats de Višik sur les problèmes elliptiques semi-linéaires par les méthodes de Minty et Browder. Bull. Soc. Math. France 93 (1965) 97-107.

[23] E.M. Stein, Singular Integrals and Differentiability Properties of Functions. Princetown University Press (1970).

[24] A. Ženíšek, The finite element method for nonlinear elliptic equations with discontinuous coefficients. Numer. Math. 58 (1990) $51-77$. 